OAK RIDGE
NATIONAL LABORATORY

MANAGED BY UT-BATTELLE

FOR THE DEPARTMENT OF ENERGY
ORNL/TM-2008/193

\section{Data Compilation for AGR-3/4 Designed-to-Fail (DTF) Fuel Particle Batch LEU04-02DTF}

\author{
John D. Hunn and James H. Miller
}

\section{October 2009}

This report was prepared as an account of work sponsored by an agency of the United States Government. Neither the United States Government nor any agency thereof, or any of their employees, makes any warranty, expressed or implied, or assumes any legal liability or responsibility for any third party's use, or the results of such use, of any information, apparatus, product or process disclosed in this report, or represents that its use by such third party would not infringe privately owned rights. 


\section{DOCUMENT AVAILABILITY}

Reports produced after January 1, 1996, are generally available free via the U.S. Department of Energy (DOE) Information Bridge.

Web site http://www.osti.gov/bridge

Reports produced before January 1, 1996, may be purchased by members of the public from the following source.

National Technical Information Service

5285 Port Royal Road

Springfield, VA 22161

Telephone 703-605-6000 (1-800-553-6847)

TDD 703-487-4639

Fax 703-605-6900

E-mail info@ntis.fedworld.gov

Web site http://www.ntis.gov/support/ordernowabout.htm

Reports are available to DOE employees, DOE contractors, Energy Technology Data Exchange (ETDE) representatives, and International Nuclear Information System (INIS)

representatives from the following source.

Office of Scientific and Technical Information

P.O. Box 62

Oak Ridge, TN 37831

Telephone 865-576-8401

Fax 865-576-5728

E-mail reports@adonis.osti.gov

Web site http://www.osti.gov/contact.html

This report was prepared as an account of work sponsored by an agency of the United States Government. Neither the United States Government nor any agency thereof, nor any of their employees, makes any warranty, express or implied, or assumes any legal liability or responsibility for the accuracy, completeness, or usefulness of any information, apparatus, product, or process disclosed, or represents that its use would not infringe privately owned rights. Reference herein to any specific commercial product, process, or service by trade name, trademark, manufacturer, or otherwise, does not necessarily constitute or imply its endorsement, recommendation, or favoring by the United States Government or any agency thereof. The views and opinions of authors expressed herein do not necessarily state or reflect those of the United States Government or any agency thereof. 


\section{Data Compilation for AGR-3/4 Designed-to-Fail (DTF) Fuel Particle Batch LEU04-02DTF}

John D. Hunn and James H. Miller

Oak Ridge National Laboratory

This document is a compilation of coating and characterization data for the AGR-3/4 designedto-fail (DTF) particles. The DTF coating is a high density, high anisotropy pyrocarbon coating of nominal $20 \mu \mathrm{m}$ thickness that is deposited directly on the kernel. The purpose of this coating is to fail early in the irradiation, resulting in a controlled release of fission products which can be analyzed to provide data on fission product transport. A small number of DTF particles will be included with standard TRISO driver fuel particles in the AGR-3 and AGR-4 compacts.

The ORNL Coated Particle Fuel Development Laboratory 50-mm diameter fluidized bed coater was used to coat the DTF particles. The coatings were produced using procedures and process parameters that were developed in an earlier phase of the project as documented in "Summary Report on the Development of Procedures for the Fabrication of AGR-3/4 Design-to-Fail Particles," ORNL/TM-2008/161. Two coating runs were conducted using the approved coating parameters. NUCO425-06DTF was a final process qualification batch using natural enrichment uranium carbide/uranium oxide (UCO) kernels. After the qualification run, LEU04-02DTF was produced using low enriched UCO kernels.

Both runs were inspected and determined to meet the specifications for DTF particles in section 5 of the AGR-3 \& 4 Fuel Product Specification (EDF-6638, Rev.1). Table 1 provides a summary of key properties of the DTF layer. For comparison purposes, an archive sample of DTF particles produced by General Atomics was characterized using identical methods. This data is also summarized in Table 1.

Table 1. Summary of DTF Coating Properties

\begin{tabular}{|l|c|c|c|c|}
\hline \multicolumn{2}{|c|}{ Specified Parameters } & NUCO425-06DTF & LEU04-02DTF & GA \\
\hline Mean Coating Thickness $(\mu \mathrm{m})$ & $20 \pm 5$ & 23.4 & 20.9 & 20.2 \\
\hline Mean Deposition Rate $(\mu \mathrm{m} / \mathrm{min})$. & $\sim 0.19$ & 0.195 & 0.190 & - \\
\hline Sink/Float Coating Density $(\mathrm{g} / \mathrm{cc})$ & $1.95 \pm 0.05$ & 1.928 & 1.937 & 1.944 \\
\hline Anisotropy (BAFo equivalent) & Not specified & 1.391 & 1.428 & 1.250 \\
\hline
\end{tabular}




\section{$\underline{\text { Table of Contents }}$}

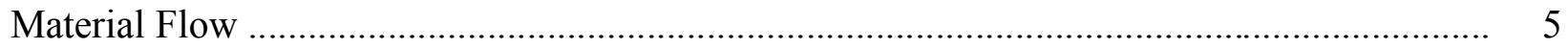

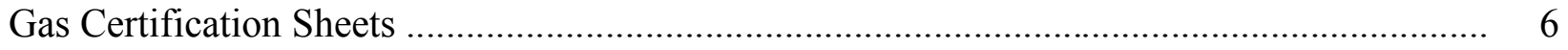

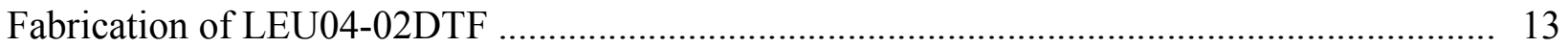

QC Acceptance Test of LEU04-02DTF ................................................................. 16

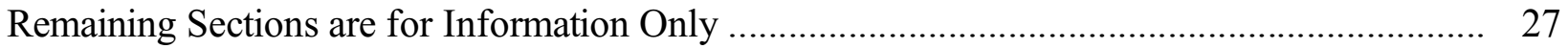

Anomalies observed during inspection by optical microscopy .................................... 28

Effect of layer compression on mercury porosimeter measurements................................... 35

Fabrication of NUCO425-06DTF .............................................................................

QC Acceptance Test of NUCO425-06DTF .............................................................. 44

Characterization of GA Archive DTF Particles .................................................................. 53 


\section{Material Flow}

Low enrichment uranium carbide/uranium oxide (UCO) used for fuel particle batch

B\&W identification : two samples NP-B8039 and NP-B8040 from composite G73I-14-69307

Two samples combined and renamed by ORNL : LEU04

Riffled sub-sample from LEU04 for coating : LEU04-02K

Renamed after coating : LEU04-02DTF

Natural enrichment uranium carbide/uranium oxide (UCO) used for qualification batch

B\&W identification : sample NP-B7869 from composite G73Z-NU-69306

Renamed by ORNL : NUCO425

Riffled sub-sample from NUCO425 for coating : NUCO425-06K

Renamed after coating : NUCO425-06DTF 


\section{$\underline{\text { Gas Certification Sheets }}$}

This section contains copies of the certificates of analysis for the gases used to coat LEU0402DTF and NUCO425-06DTF. Table 2 lists the cylinders used for each gas.

Table 2. Cylinder number(s) of gases used to produce DTF batches

\begin{tabular}{|l|l|l|l|}
\hline Batch ID & Propylene & Helium & Argon \\
\hline NUCO425-06DTF & $9198633 \mathrm{G}$ & XGO00227/XGO00103 & BM104495/F04959/EL22118 \\
\hline LEU04-02DTF & $9198633 G$ & XGO00227/XGO00103 & BM104495/F04959/EL22118 \\
\hline
\end{tabular}


ORNL/TM-2008/193

$16 \mid$

AIR LIQUIDE

$-T$

Customer :A L A C Oakridge

P.O. \# :072302 Doo:10685248-1A

Blend Type : LIQUID CERTIFIED

Cyl. Size :22LP Item \#:

Cert. Date : :7-25-2002 VaK : CGA 510

Cylinder \# : 91986336

8992 grams

Mole Component

Balance PROPYLENE CP GRADE IMPURITIES:

$\because \quad .403 \%$ PROPANF

$\therefore \quad$ Valid Lntil:

24 July, 2004

$$
\begin{array}{r}
\text { NVCOUZS- } \\
\text { OZDOTF } \\
\text { O3OTF } \\
\text { OUDTF } \\
\text { OSDTF } \\
\text { O6DTF } \\
\text { LEVOU-O2DTF }
\end{array}
$$

funsts. Millas 10-7-08 


\section{CERTIFICATE OF BATCH/LOT ANALYSIS , JG $/ / 108$}

Certification Of Batch/Lot \#: 169A Ua.8625A

Product: Helium

Customer:

Date of Certification:

P.O. Number:

Document Number:

OAKRIDGE

6/10/2008
Grade: Ultra High Purity

Test Cylinder \#: $12 / \mathrm{pk}$

Item Number:

Valve: $\quad 580$

Cylinder Size: $\quad 44 / 12$

\section{ANALYSIS REPORT}

Major Component

Helium Ultra High Purity

$\frac{\text { Specification }}{99.9990 \%}$

$99.9990 \%$

Notes:

\begin{tabular}{|c|}
\hline Impurities \\
\hline Moisture \\
\hline Oxygen \\
\hline
\end{tabular}

Total Hydrocarbons

\begin{tabular}{c} 
Specification \\
\hline$<3 \mathrm{ppm}$ \\
$<2 \mathrm{ppm}$ \\
$<0.5 \mathrm{ppm}$
\end{tabular}

Cyliadre It XGO\$O227

Nuco425-34DTF

$$
\begin{gathered}
08 D T F \\
00 D T \\
\text { HEVU-O2DTF }
\end{gathered}
$$

\begin{tabular}{c} 
Actual Analysis \\
\hline $0.4 \mathrm{PPM}$ \\
$0.9 \mathrm{PPM}$ \\
N/D
\end{tabular} $\frac{\text { Purity }}{>99.9990 \%}$
Air Liquide America, L.P.

ICSC-CYL.0307-W
1311 New Savannah Rd. Augusta, GA $30901-3843$

Phone: 706-724-8725

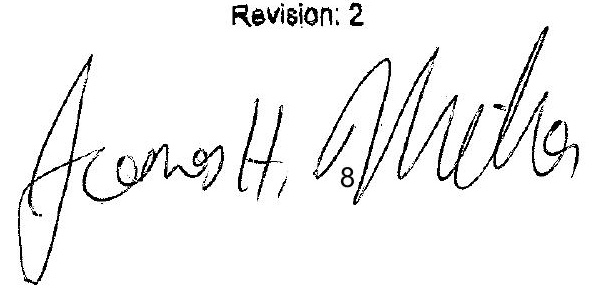

$60+2)$ 

$5^{8}$
instellei in poralled h/ ather the 9-10-08
ORNL/TM-2008/193

AIR LIOUIDE

CERTIFICATE OF BATCH/LOT ANALYSIS

Certification Of Batch/Lot \#:

137AUG8628A

Product: Helium

Grade: Ultra High Purity

Customer: UTBathelle

Date of Certification: 3 .Jun

P.O. Number: 4800492868

Document Number: 30650563
Test Cylinder \#: X535

Item Number: $\quad 0030 \mathrm{~A}-3000$

Valve: $\quad 580$

Cylinder Size: 44

\section{ANALYSIS REPORT}

$\frac{\text { Major Component }}{\text { Helium Ultra High Purity }}$
$\frac{\text { Impurities }}{\text { Moisture }}$
Oxygen

Total Hydrocarbons

\begin{tabular}{cc} 
Specification & Purity \\
\hline $99.9990 \%$ & $>99.999 \%$
\end{tabular}

Actual Analysis

$0.2 \mathrm{ppm}$

$0.5 \mathrm{ppm}$

$0.1 \mathrm{ppm}$

$<0.5 \mathrm{ppm}$

Notes:

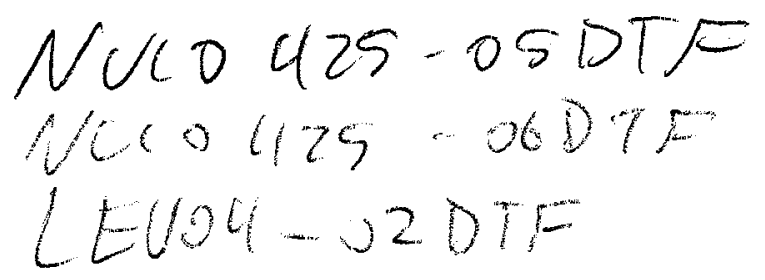

Certified By:
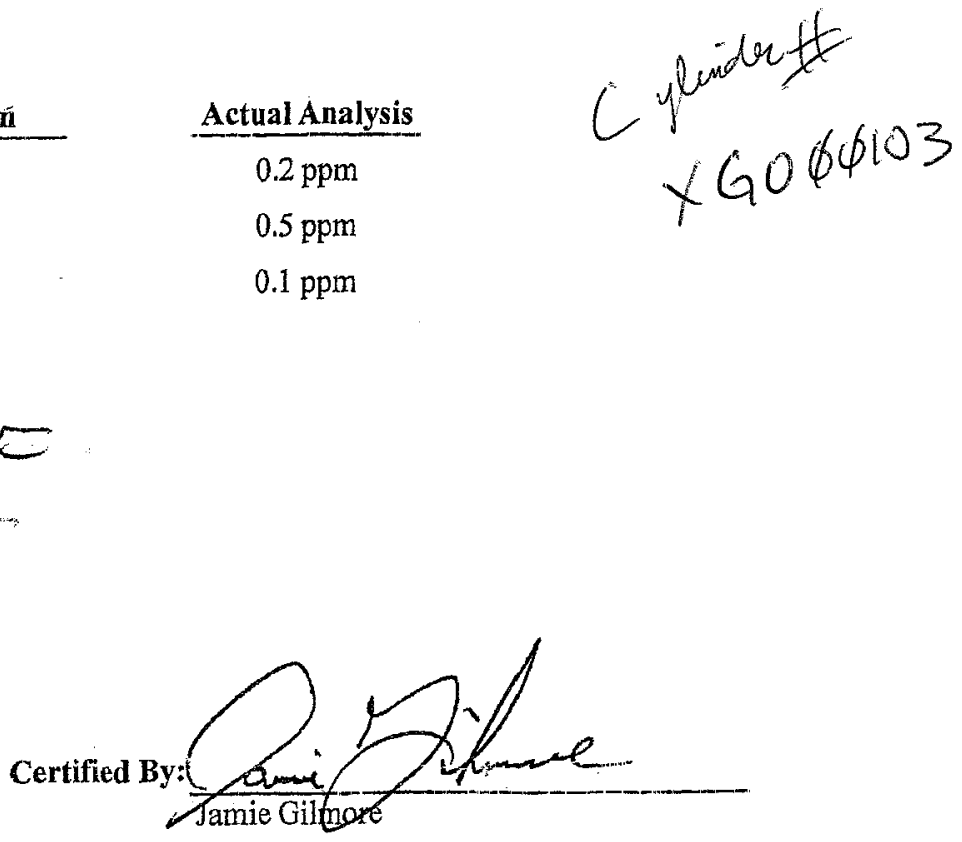

Air Liquide America, L.P.

1001 Alvin Weinberg Dr. Oakridge, TN 37830-8012

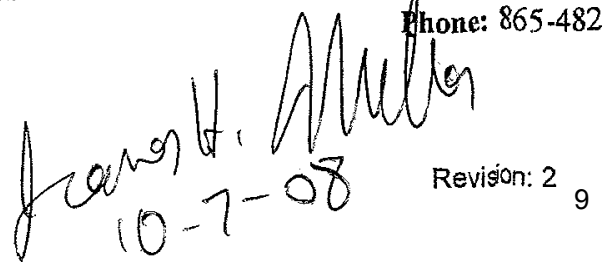


2

\section{CERTIFICATE OF ANALYSIS}

\section{Certification Of Cylinder \#: $\quad$ BM104495}

Product: Argon

Grade: Ultra High Purity

\begin{tabular}{llll} 
Customer: & UT Battelle & Batch/Lot \#: & 2160 RG8395A \\
Date of Certification: & $8 / 14 / 2008$ & Item Number: & $0013-1300$ \\
P.O. Number: & 4800491160 & Valve: & 580 \\
Document Number: & 3058828 & Cylinder Size: & 44 \\
\hline
\end{tabular}

\section{ANALYSIS REPORT}

\section{Major Component}

Argon Ultra High Purity

Impurities
Moisture
Oxygen
Total Hydrocarbons

Notes:

$\frac{\text { Specification }}{99.9990 \%} \quad-\frac{\text { Purity }}{>99.999 \%}$

\begin{tabular}{cc} 
Specification & Actual Analysis \\
\cline { 1 - 2 }$<3 \mathrm{ppm}$ & $0.2 \mathrm{ppm}$ \\
$<2 \mathrm{ppm}$ & $1.1 \mathrm{ppm}$ \\
$<0.5 \mathrm{ppm}$ & $0.1 \mathrm{ppm}$
\end{tabular}

$$
\begin{aligned}
& \text { NUEO42S-O4DTF } \\
& -05015 \\
& 60 D T \\
& \angle E C 04 \cdot 020 T A
\end{aligned}
$$

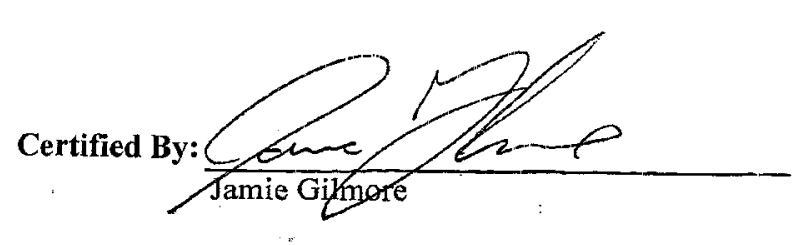

Air Liquide America, L.P.

1001 Alvin Weinberg Dr. Oakridge, TN 37830-8012

Phone: 865-482-7046 


\section{CERTIFICATE OF ANALYSIS}

Certification Of Cylinder \#:

F04959

Product: Argon

Grade: Ultra High Purity

\begin{tabular}{|c|c|c|c|}
\hline Customer: & UT Battelle & Batch/Lot \#: & 210ORG8395A \\
\hline Date of Certification: & $8 / 14 / 2008$ & Item Number: & $0013-1300$ \\
\hline P.O. Number: & 4800491160 & Valve: & 580 \\
\hline Document Number: & 3058828 & Cylinder Size: & 44 \\
\hline
\end{tabular}

\section{ANALYSIS REPORT}

Major Component

Argon Ultra High Purity

$\frac{\text { Specification }}{99.9990 \%}$

$99.9990 \%$
Purity

$>99.999 \%$

\begin{tabular}{|c|}
\hline Impurities \\
\hline Moisture \\
\hline Oxygen \\
\hline
\end{tabular}

Total Hydrocarbons

\begin{tabular}{c} 
Specification \\
\hline $3 \mathrm{ppm}$ \\
$<2 \mathrm{ppm}$ \\
$<0.5 \mathrm{ppm}$
\end{tabular}

Specification

Actual Analysis
$0.2 \mathrm{ppm}$
$1.3 \mathrm{ppm}$
$0.1 \mathrm{ppm}$

$$
\begin{aligned}
& \text { O5 DTF } \\
& \text { NUCU425-05DTF } \\
& \text { O6 DTE } \\
& \text { LEVO4-02DTF }
\end{aligned}
$$

Notes:

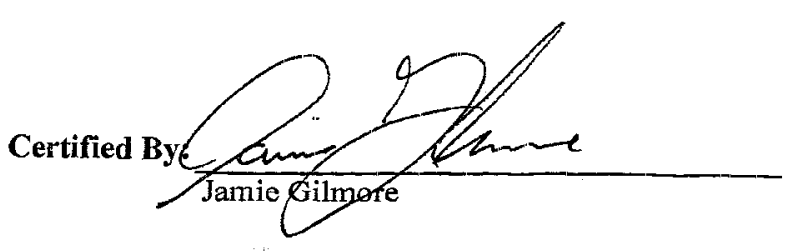

Air Liquide America, L.P.

1001 Alvin Weinberg Dr. Oakridge, TN 37830-8012

Phone: 865-482-7046 
ORNL/TM-2008/193

AIR LIQUID

Bar Code HA 31192A9701

CERTIFICATE OF ANALYSIS

\begin{tabular}{llll} 
& $\begin{array}{c}\text { Certification Of Cylinder \#: } \\
\text { Product: Argon }\end{array}$ & $\begin{array}{l}\text { EL22118 } \\
\text { Grade: }\end{array}$ & $\begin{array}{c}\text { Ultra High Purity } \\
\text { 9-19-08 }\end{array}$ \\
\hline Customer: & UT Battelle & Batch/Lot \#: & 2160 GG8395A \\
Date of Certification: & $8 / 14 / 2008$ & Item Number: & $0013-1300$ \\
P.O. Number: & 4800491160 & Valve: & 580 \\
Document Number: & 3058828 & Cylinder Size: 44 \\
\hline
\end{tabular}

ANALYSIS REPORT

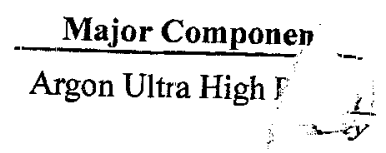

\begin{tabular}{c} 
Impurities \\
\hline Moisture \\
Oxygen \\
Total Hydrocarbons
\end{tabular}

$$
\begin{aligned}
& \text { NCCO425-06DTF } \\
& \text { LEU O4-O2DTF }
\end{aligned}
$$

$\frac{\text { Specification }}{99.9990 \%}$

\begin{tabular}{c} 
Specification \\
\hline$<3 \mathrm{ppm}$ \\
$<2 \mathrm{ppm}$ \\
$<0.5 \mathrm{ppm}$
\end{tabular}

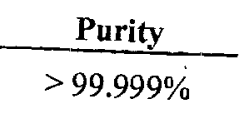

Actual Analysis

$0.2 \mathrm{ppm}$

$1.1 \mathrm{ppm}$

$0.1 \mathrm{ppm}$

Notes:

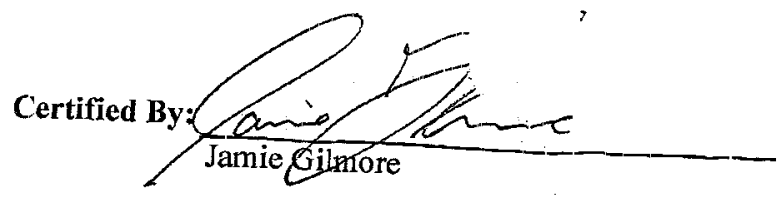

Air Liquide America, L.P.

1001 Alvin Weinberg Dr. Oakridge, TN 37830-8012

Phone: 865-482-7046

ICSC-CYL-0307-W

Revision: 2

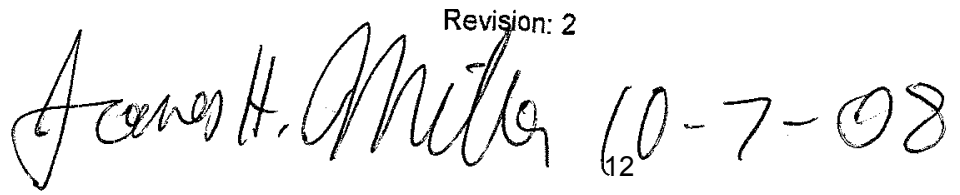

Effect' 


\section{Fabrication of LEU04-02DTF}

ORNL AGR program coating procedure AGR-DTF-COAT-SOP-01 was used to fabricate a DTF coating on low enrichment UCO kernels using the ORNL Coated Particle Fuel Development Laboratory $50-\mathrm{mm}$-diameter fluidized bed coater. Table 3 gives a summary of the process conditions and the resulting properties of the DTF coated particles. The coating process conditions met the specifications for DTF particles in section 3.2 of the AGR-3 \& 4 Fuel Product Specification (EDF-6638, Rev.1), with the exception of the coating gas fraction (CGF), for which a deviation request was approved (see note 1 below). A copy of the coating summary sheet from the laboratory log book is included in this section.

Table 3. Summary of DTF Coating Conditions and Results

\begin{tabular}{|c|c|c|}
\hline Parameter & Specified Value & LEU04-02DTF \\
\hline He $(\mathrm{sccm})$ & Not specified & 12,200 \\
\hline Propylene $(\mathrm{sccm})$ & Not specified & 130 \\
\hline TGF $(\mathrm{sccm})$ & Not specified & 12,330 \\
\hline CGF & $0.011 \pm 0.002$ (note 1$)$ & 0.0105 \\
\hline Coating Temperature $\left({ }^{\circ} \mathrm{C}\right)$ & $1285 \pm 25$ & 1260.7 \\
\hline Coating Time $(\mathrm{min})$. & Not specified & 110 \\
\hline Coating Thickness $(\mu \mathrm{m})$ & $20 \pm 5$ & 20.9 \\
\hline Deposition Rate $(\mu \mathrm{m} / \mathrm{min})$. & $\sim 0.19$ & 0.190 \\
\hline Coating Density $(\mathrm{g} / \mathrm{cc})$ & $1.95 \pm 0.05$ & 1.937 \\
\hline Anisotropy $($ BAFo equiv. $)$ & Not specified & 1.428 \\
\hline
\end{tabular}

Note 1. The coating gas fraction specification in the AGR-3 \& 4 Fuel Product Specification (EDF-6638, Rev.1) was $0.015 \pm 0.0015$. A deviation request (DR-ORNL-AGR-08-01) to operate in the region $0.011 \pm 0.002$ was approved. This region was determined during development efforts to produce coatings of the desired properties. A copy of the approved deviation request is included in this section. 
Standard Operating Procedure

Fluidized Bed Chemical Vapor Deposition System - DTF

APPENDIX $K:$ COATING SUMMARY

\begin{tabular}{|c|c|c|c|c|}
\hline \multicolumn{2}{|c|}{ Coating Run No. } & \multicolumn{3}{|c|}{$\angle E 004-02 D T E$} \\
\hline \multicolumn{2}{|c|}{ Description: } & \multicolumn{3}{|c|}{ DTF particles } \\
\hline \multicolumn{2}{|c|}{ Kernel Lot No. } & \multicolumn{3}{|c|}{$\angle E V O A-02 K$} \\
\hline \multicolumn{2}{|c|}{ Kernel Composition } & \multicolumn{3}{|c|}{$\angle E V C O$} \\
\hline \multicolumn{5}{|c|}{ Kernel Diameter $(\mu \mathrm{m})$} \\
\hline \multicolumn{5}{|c|}{ Kernel Density $\left(\mathrm{g} / \mathrm{cm}^{3}\right)$} \\
\hline \multicolumn{2}{|c|}{ Kernel Batch Wt. (g) } & \multicolumn{3}{|l|}{76.4326} \\
\hline \multicolumn{5}{|c|}{ Kernel surface area $\left(\mathrm{cm}^{2}\right)$} \\
\hline \multicolumn{5}{|c|}{ Kernel volume $\left(\mathrm{cm}^{3}\right)$} \\
\hline \multicolumn{5}{|c|}{ After Coating } \\
\hline \multicolumn{2}{|c|}{ Coated Particle Batch Wt. (g) } & \multicolumn{3}{|c|}{86.9326979 .0566} \\
\hline \multirow{2}{*}{\multicolumn{2}{|c|}{ Coating Wt. (g) }} & \multicolumn{3}{|c|}{2.1240} \\
\hline & & DTF Paramete & & As-Processed \\
\hline \multicolumn{5}{|c|}{ Carbon } \\
\hline \multicolumn{2}{|c|}{ Coating gases } & \multicolumn{2}{|l|}{$\mathrm{He}+\mathrm{C}_{3} \mathrm{H}_{6}$} & $4 c+C_{3} H_{6}$ \\
\hline \multicolumn{2}{|c|}{ TGF } & \multicolumn{2}{|l|}{ Not specified } & 12330 \\
\hline \multicolumn{2}{|l|}{ CGF } & \multicolumn{2}{|l|}{$0.015 \pm 0.0015$} & 0.0105 \\
\hline \multicolumn{2}{|l|}{ CGR } & \multicolumn{2}{|l|}{1 (Propylene only) } & $c$ \\
\hline \multicolumn{2}{|c|}{ Temperature } & \multicolumn{2}{|l|}{$1285 \pm 25^{\circ} \mathrm{C}$} & 1260 \\
\hline \multicolumn{2}{|c|}{ Helium flow rate $\left(\mathrm{cm}^{3} / \mathrm{min}\right)$} & \multicolumn{2}{|l|}{ Not specified } & 12200 \\
\hline Propylene flc & $w$ rate $\left(\mathrm{cm}^{3} / \mathrm{min}\right)$ & Not specified & & 130 \\
\hline Time & & Not specified & & 110 \\
\hline Coating rate & $\mu \mathrm{m} / \mathrm{min})$ & $\sim 0.19$ & & 0.19 \\
\hline Coating thick & ess $(\mu \mathrm{m})$ & $20 \pm 5$ & & 20.9 \\
\hline Coating Den & ity $\left(\mathrm{g} / \mathrm{cm}^{3}\right)$ & $1.95 \pm 0.05$ & & 1.9372 \\
\hline Comments $/ \mathrm{N}$ & otes: & & & \\
\hline Operator: & termett. & When & Date: & $10-23-08$ \\
\hline Verified by: & & $\alpha i$ & Date: & $10 / 23 / 08$ \\
\hline QAS: & & & Date: & $10 / 27 / 08$ \\
\hline
\end{tabular}




\begin{tabular}{|c|c|c|}
\hline \multicolumn{2}{|l|}{$\begin{array}{l}\text { 1. DEVIATION NO: } \\
\text { DR- ORNL-AGR-08-01 }\end{array}$} & 2. DIVISIO:V: Materials Science\&Tech. \\
\hline \multicolumn{2}{|l|}{$\begin{array}{l}\text { 3. PROJECT TITLE: } \\
\text { N/A }\end{array}$} & $\begin{array}{l}\text { 4. PROGRAM: } \\
\text { Advanced Gas Reactor Fuel Developritit ! and Qualification }\end{array}$ \\
\hline $\begin{array}{l}\text { 5. ITEM/ACTIVITY NAME: } \\
\text { DTF Coating for AGR-3/4 }\end{array}$ & & $\begin{array}{l}\text { 6. SPECIFICATION/PROCEDURE: } \\
\text { AGR Program/NL Specilication \#EDF.66: } 38 \text {, Revision } 1\end{array}$ \\
\hline $\begin{array}{l}\text { 7. DRAWING NO: } \\
\text { N/A }\end{array}$ & $\begin{array}{l}\text { 8. SHOP ORDER: } \\
\text { N/A }\end{array}$ & $\begin{array}{l}\text { 9. WORK/PU]:LCHASE: } \\
\text { N/A }\end{array}$ \\
\hline \multicolumn{3}{|c|}{ 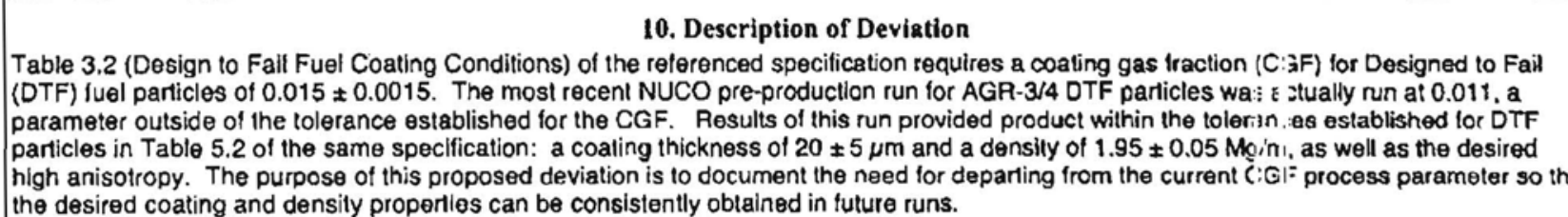 } \\
\hline
\end{tabular}

the desired coating and density properlies can be consistently oblained in future runs.

\section{Justification and Limitations}

Though the CGF of NUCO425-05DTF did not fall within the process condition tolerance provided in Table 3.2 , thi) ( 2 ating process is reproducible and has demonstrated the ability to produce particles with the desired coating thickness and density e: tablished in Table 5.2 . Further coating development in an attempt to adhere to process conditions within the specification that produce far icles thal meet the product specification is not thought to be feasible, and is therefore not recommended.

It is recommended that the CGF process parameter of $0.011 \pm 0.002$ be used in the planned NUCO verilication rut1, : nd in subsequent runs to produce the LEUCO DTF particles.

12. Requested by: J. H. Miller

13. Title: Change to DTF Coating Gas Fraction

15. Drawing/Document is to be revised $\square$ NO $\square$ YES (If yes, list):

\begin{tabular}{|l|l|l|l|l|}
\hline Approved by: & $\begin{array}{l}\text { 16. Task Leader: } \\
\text { J. D. Hunn }\end{array}$ & Date: \\
\cline { 2 - 5 } As Appropriatc & $\begin{array}{l}\text { 17. Requirement Originator: } \\
\text { INL Rep. }\end{array}$ & $\begin{array}{l}\text { Date: } \\
9-22-08\end{array}$ & $\begin{array}{l}\text { 19. Item User: } \\
\text { N/A }\end{array}$ \\
\cline { 2 - 5 } & $\begin{array}{l}\text { 18. QA Group } \\
\text { M. C. Vance }\end{array}$ & $\begin{array}{l}\text { 20. Other: } \\
\text { N/A }\end{array}$ & Date: \\
\hline
\end{tabular}

ORNI_313/Page I of 3 (4-2003) 


\section{QC Acceptance Test of LEU04-02DTF}

Product inspection plan AGR-CHAR-PIP-08 was used to characterize the LEU04-02DTF particle batch. This section contains the inspection report form (IRF-08) and associated data report forms resulting from that analysis. The LEU04-02DTF particle batch was found to meet the specifications for DTF particles in section 5 of the AGR-3 \& 4 Fuel Product Specification (EDF-6638, Rev.1).

Open porosity, which was specified for measurement only, could not be determined due to compression of the DTF coating. This is noted in DRF-31 and described in detail in the section "Effect of layer compression on mercury porosimeter measurements". 
ORNL/TM-2008/193

Inspection Report Form IRF-08: AGR-3/4 Designed to Fail Coated Particle Batches

Procedure: AGR-CHAR-PIP-08 Rev. 0

Coated particle batch ID: LEU 04-02DTF

Coated particle batch description: AGR-3/4 DTF Particle Batch

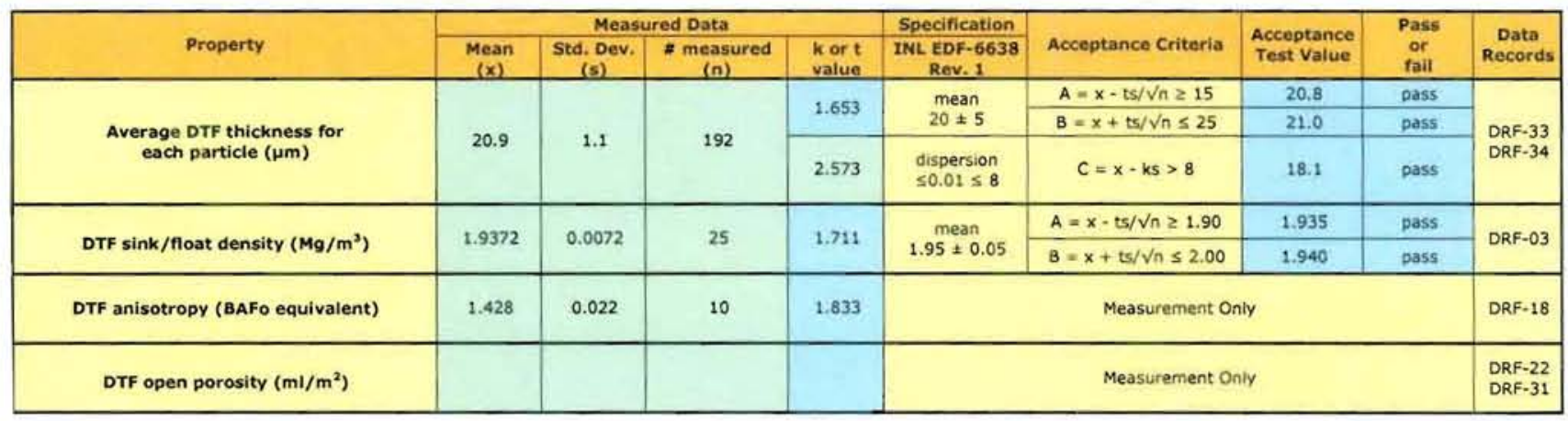

Open porosity value unavailable due to compression of OTF layer.

Comments

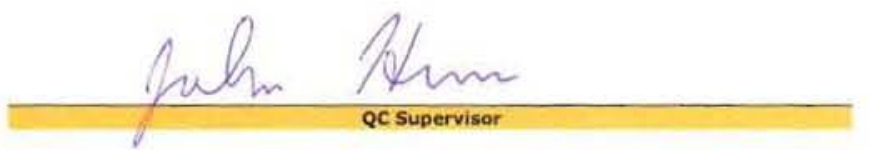

Accept coated particle batch (Yes or No):

Yes

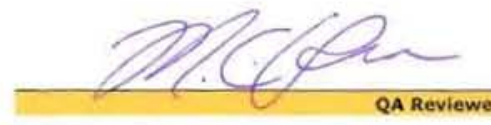

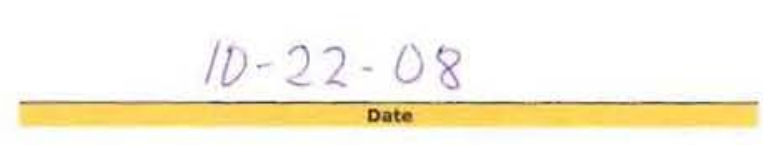

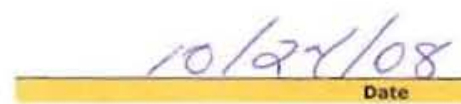

17 
Data Report Form DRF-33: Imacina of Small Particle Cross-sections Usina an Ootical Microscope System

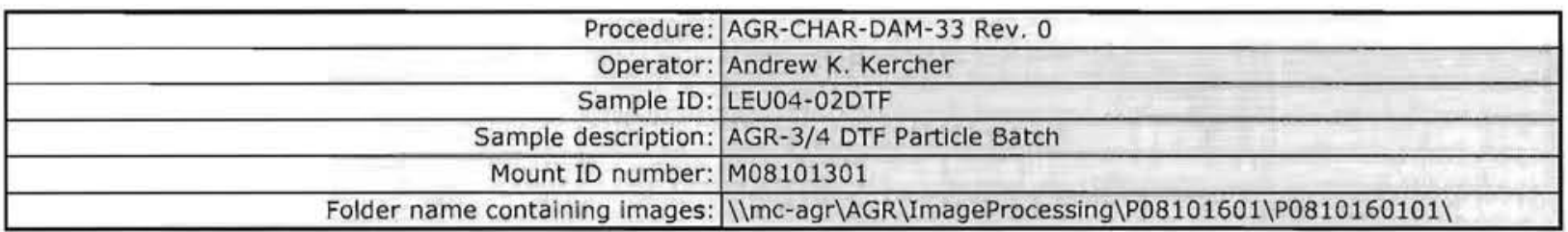

\begin{tabular}{|r|r|}
\hline DMR calibration expiration date: & $9 / 15 / 2009$ \\
\hline Calibrated pixels/micron: & 4.4833 \\
\hline Stage micrometer calibration expiration date: & $2 / 13 / 2009$ \\
\hline Measured value for $300 \mu \mathrm{m}$ in stage micrometer image $(\mu \mathrm{m}):$ & 300.0 \\
\hline
\end{tabular}

\begin{tabular}{|c|c|c|c|c|c|c|c|c|}
\hline \multicolumn{5}{|c|}{ Polish-down distance $n, m(\mu \mathrm{m})$} \\
\hline 2,2 & 2,8 & 8,2 & 8,8 \\
\hline \hline 174 & 179 & 184 & 188 \\
\hline
\end{tabular}
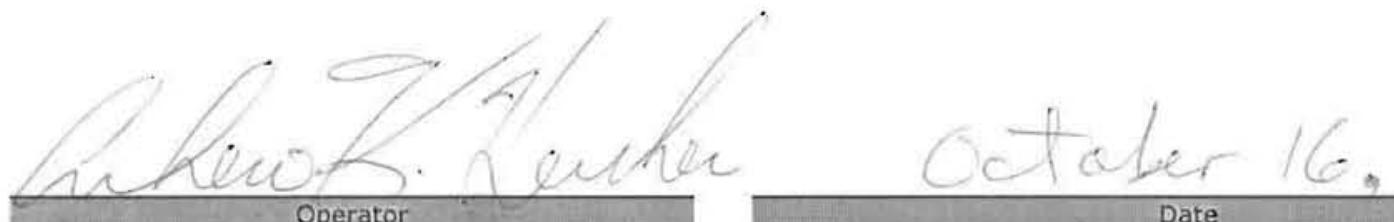
Data Report Form DRF-33: Imaaino of Small Particle Cross-sections Using an Optical Microscope System

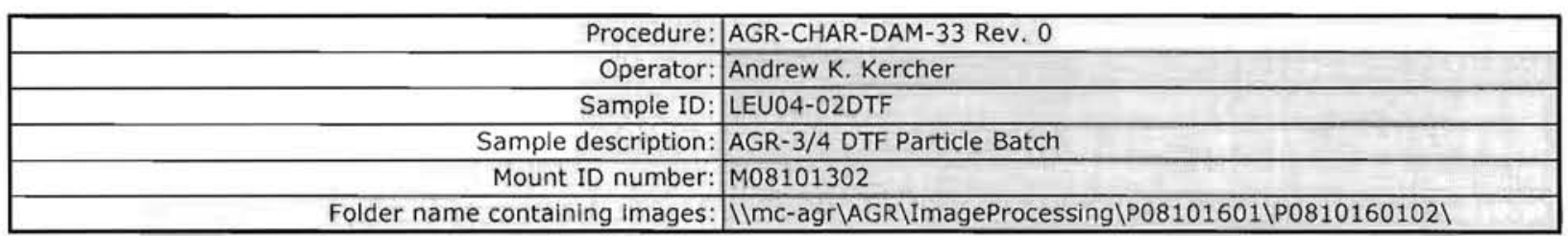

\begin{tabular}{|r|l|}
\hline DMR calibration expiration date: & $9 / 15 / 2009$ \\
\hline Calibrated pixels/micron: & 4.4833 \\
\hline Stage micrometer calibration expiration date: & $2 / 13 / 2009$ \\
\hline Measured value for $300 \mu \mathrm{m}$ in stage micrometer image $(\mu \mathrm{m}):$ & 300.0 \\
\hline
\end{tabular}

\begin{tabular}{|c|c|c|c|}
\hline \multicolumn{4}{|c|}{ Polish-down distance $\mathrm{n}, \mathrm{m}(\mu \mathrm{m})$} \\
\hline 2,2 & 2,8 & 8,2 & 8,8 \\
\hline \hline 171 & 175 & 171 & 174 \\
\hline
\end{tabular}

\begin{tabular}{|c|c|c|c|c|}
\hline \multicolumn{5}{|c|}{ Approximate layer width in polish plane $(\mu \mathrm{m})$} \\
\hline Kernel radius & Layer 1 & Layer 2 & Layer 3 & Layer 4 \\
\hline \hline 202 & 20 & & & \\
\hline
\end{tabular}
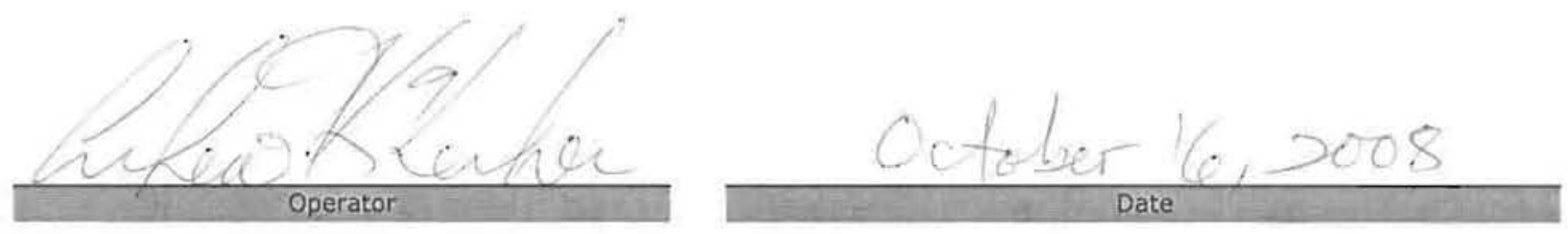
Data Report Form DRF-33: Imaaing of Small Particle Cross-sections Using an Optical Microscope System

\begin{tabular}{|r|l|}
\hline Procedure: & AGR-CHAR-DAM-33 Rev. 0 \\
\hline Operator: & Andrew K. Kercher \\
\hline Sample ID: & LEU04-02DTF \\
\hline Sample description: & AGR-3/4 DTF Particle Batch \\
\hline Mount ID number: & M08101303 \\
\hline Folder name containing images: & IIMc-agr\AGR ImageProcessing \P08101601\P0810160103\} $\\
{\hline}$
\end{tabular}

DMR calibration expiration date: $9 / 15 / 2009$

Calibrated pixels/micron: 4.4833

Stage micrometer calibration expiration date: 2/13/2009

Measured value for $300 \mu \mathrm{m}$ in stage micrometer image $(\mu \mathrm{m}): 300.0$

\begin{tabular}{|c|c|c|c|}
\hline \multicolumn{4}{|c|}{ Polish-down distance $n, m(\mu \mathrm{m})$} \\
\hline 2,2 & 2,8 & 8,2 & 8,8 \\
\hline \hline 183 & 194 & 182 & 191 \\
\hline
\end{tabular}

Approximate layer width in polish plane $(\mu \mathrm{m})$

\begin{tabular}{|l|l|l|l|l|}
\hline Kernel radius & Layer 1 & Layer 2 & Layer 3 & Layer 4 \\
\hline
\end{tabular}

\begin{tabular}{l|l}
209 & 20 \\
\hline
\end{tabular}
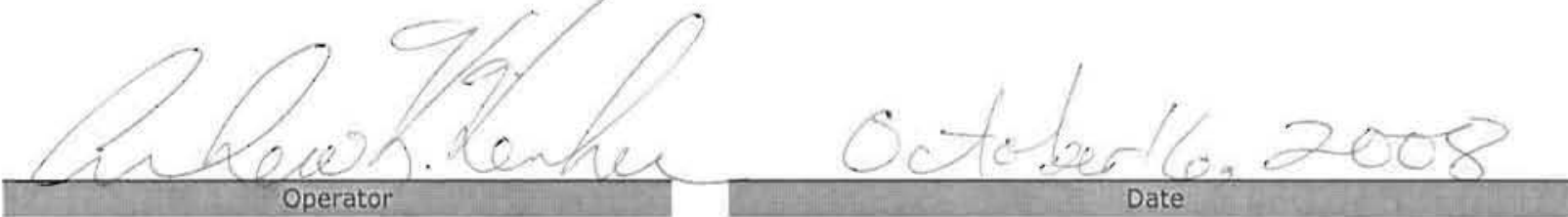

Date 


\begin{tabular}{|c|c|}
\hline Procedure: & AGR-CHAR-DAM-34 ReV. 0 \\
\hline Operator: & Andrew K. Kercher \\
\hline Folder name containing images: & 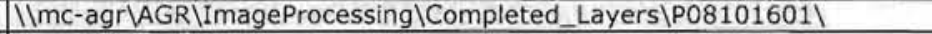 \\
\hline Sample ID: & LEU04-02DTF \\
\hline Sample Description: & AGR-3/4 DTF Particle Batch \\
\hline containing processed data: & IImc-agr|AGR\ImageProcessing\Comp \\
\hline
\end{tabular}

Folder name containing processed data: IImc-agr|AGR\ImageProcessing|Completed_Layers\P08101601_output|

\begin{tabular}{|r|c|}
\hline Number of layers analyzed: & 192 \\
\hline Mean of the average layer 1 thickness of each particle $(\mu \mathrm{m}):$ & 20.9 \\
\hline Standard deviation in the average layer 1 thickness of each particle $(\mu \mathrm{m}):$ & 1.1 \\
\hline
\end{tabular}

\section{Distribution of the average laver 1 thickness (top binned)}

\begin{tabular}{|c|c|}
\hline Layer 1 Thickness $(\mu \mathrm{m})$ & Frequency \\
\hline \hline$<12$ & 0 \\
\hline 13 & 0 \\
\hline 14 & 0 \\
\hline 15 & 0 \\
\hline 16 & 0 \\
\hline 17 & 2 \\
\hline 18 & 1 \\
\hline 19 & 4 \\
\hline 20 & 20 \\
\hline 21 & 74 \\
\hline 22 & 65 \\
\hline 23 & 21 \\
\hline 24 & 4 \\
\hline 25 & 0 \\
\hline 26 & 1 \\
\hline$>26$ & 0 \\
\hline & \\
\hline
\end{tabular}

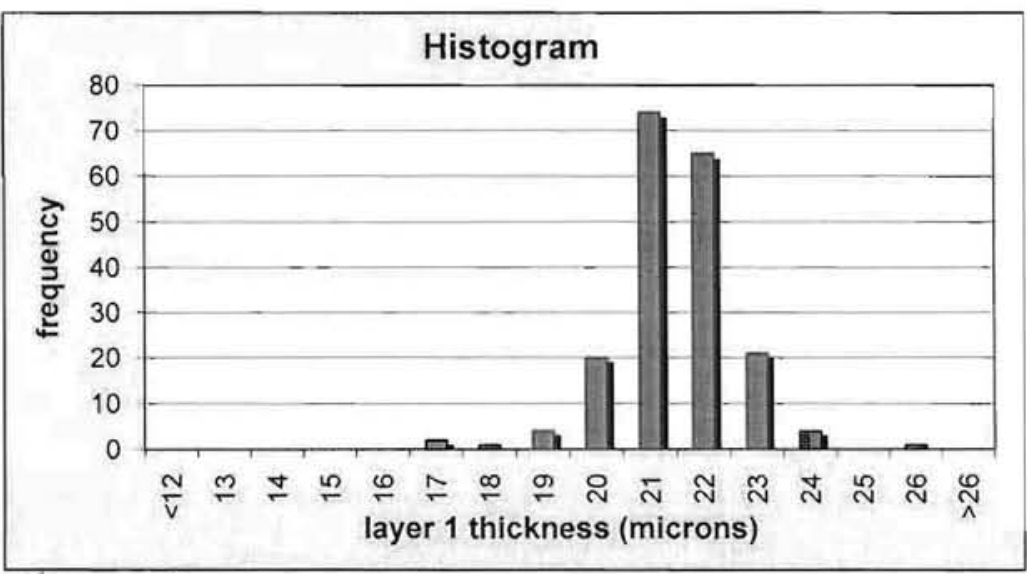

Operator

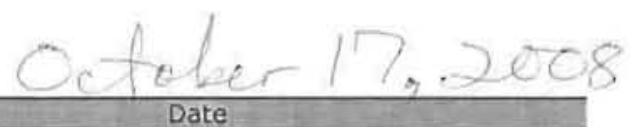




\section{Data Report Form DRF-03: Measurement of PyC Density usinq a Density Gradient Column}

\begin{tabular}{|c|c|}
\hline Procedure: & AGR-CHAR-DAM-03 Rev. 3 \\
\hline Operator: & Dixie Barker \\
\hline Filename: & IImc-agr\AGR\DensityColumn\D080100701_DRF03R3.xis \\
\hline Sample ID: & LEU04-02DTF-C01 \\
\hline Sample description: & AGR-3/4 DTF Particle Batch \\
\hline Float expiration date: & $07 / 2012$ \\
\hline Gauge expiration date: & $11 / 2008$ \\
\hline Bath temperature: & $23.0^{\circ} \mathrm{C}$ \\
\hline
\end{tabular}

\begin{tabular}{|c|c|c|c|}
\hline \multicolumn{5}{|c|}{ Callbrated Floats } \\
\hline Density & $\begin{array}{c}\text { Top of } \\
\text { Float }\end{array}$ & $\begin{array}{c}\text { Bottom of } \\
\text { Float }\end{array}$ & $\begin{array}{c}\text { Center of } \\
\text { Mass }\end{array}$ \\
\hline \hline 1.800 & 109.87 & 118.05 & 113.96 \\
\hline 1.850 & 218.06 & 225.78 & 221.92 \\
\hline 1.900 & 324.75 & 330.69 & 327.72 \\
\hline 1.950 & 429.02 & 435.89 & 432.46 \\
\hline 2.000 & 528.67 & 535.71 & 532.19 \\
\hline \multicolumn{5}{|c|}{ Linear Fit } \\
\hline \multicolumn{5}{|c|}{} \\
\hline Slope & StDev & intercept & StDev \\
\hline $4.77 E-04$ & $2.97 E-06$ & $1.74 E+00$ & $9.74 E-04$ \\
\hline
\end{tabular}

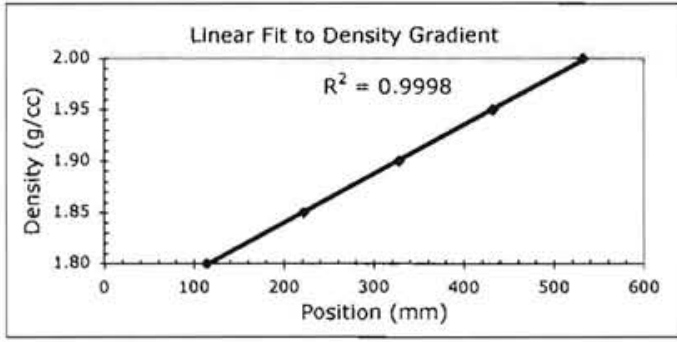

\begin{tabular}{|c|c|c|c|c|c|c|c|c|}
\hline \multicolumn{9}{|c|}{ Sample Density } \\
\hline $\begin{array}{l}\text { Fragment } \\
\text { Number }\end{array}$ & $\begin{array}{c}\text { Fragment } \\
\text { Position }\end{array}$ & $\begin{array}{c}\text { Calculated } \\
\text { Density }\end{array}$ & $\begin{array}{l}\text { Fragment } \\
\text { Number }\end{array}$ & $\begin{array}{l}\text { Fragment } \\
\text { Position }\end{array}$ & $\begin{array}{c}\text { Calculated } \\
\text { Density }\end{array}$ & $\begin{array}{l}\text { Fragment } \\
\text { Number }\end{array}$ & $\begin{array}{l}\text { Fragment } \\
\text { Position }\end{array}$ & $\begin{array}{c}\text { Calculated } \\
\text { Density }\end{array}$ \\
\hline 1 & 381.31 & 1.9266 & 26 & & & 51 & & \\
\hline 2 & 381.31 & 1.9266 & 27 & & & 52 & & \\
\hline 3 & 387.59 & 1.9296 & 28 & & & 53 & & \\
\hline 4 & 381.37 & 1.9266 & 29 & & & 54 & & \\
\hline 5 & 388.43 & 1.9300 & 30 & & & 55 & & \\
\hline 6 & 389.90 & 1.9307 & 31 & & & 56 & & \\
\hline 7 & 392.36 & 1.9319 & 32 & & & 57 & & \\
\hline 8 & 387.51 & 1.9295 & 33 & & & 58 & & \\
\hline 9 & 391.68 & 1.9315 & 34 & & & 59 & & \\
\hline 10 & 398.68 & 1.9349 & 35 & & & 60 & & \\
\hline 11 & 403.31 & 1.9371 & 36 & & & 61 & & \\
\hline 12 & 406.45 & 1.9386 & 37 & & & 62 & & \\
\hline 13 & 409.18 & 1.9399 & 38 & & & 63 & & \\
\hline 14 & 410.23 & 1.9404 & 39 & & & 64 & & \\
\hline 15 & 403.59 & 1.9372 & 40 & & & 65 & & \\
\hline 16 & 406.43 & 1.9386 & 41 & & & 66 & & \\
\hline 17 & 406.76 & 1.9387 & 42 & & & 67 & & \\
\hline 18 & 409.38 & 1.9400 & 43 & & & 68 & & \\
\hline 19 & 410.32 & 1.9404 & 44 & & & 69 & & \\
\hline 20 & 419.04 & 1.9446 & 45 & & & 70 & & \\
\hline 21 & 422.68 & 1.9463 & 46 & & & 71 & & \\
\hline 22 & 422.48 & 1.9462 & 47 & & & 72 & & \\
\hline 23 & 421.92 & 1.9460 & 48 & & & 73 & & \\
\hline 24 & 426.56 & 1.9482 & 49 & & & 74 & & \\
\hline 25 & 431.44 & 1.9505 & 50 & & & 75 & & \\
\hline & Avera & e density of & C fragments: & & & 1.9372 & & \\
\hline Stan & rd deviation & $n$ density of $\mathrm{f}$ & fracments: & & & 0.0072 & & \\
\hline Uncerta & ty in calculat & $d$ density of $F$ & C fragments: & & & 0.0016 & & \\
\hline
\end{tabular}
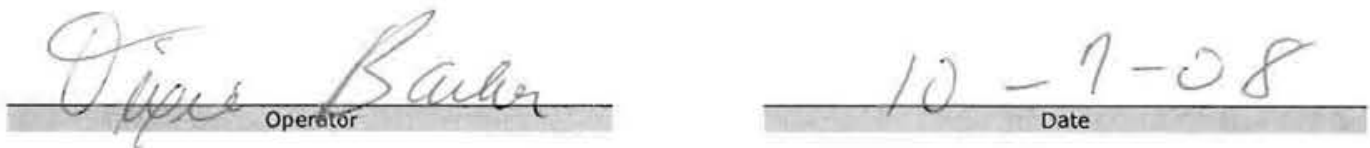
Data Report Form DRF-18A: Measurement of Pyrocarbon Anisotropy using the 2-MGEM - IPyC

\begin{tabular}{|r|l|}
\hline Procedure: & AGR-CHAR-DAM-18 Rev. 1 \\
\hline Operator: & G. E. Jellison \\
\hline Mount ID: & M08101301 \\
\hline Sample ID: & LEU04-02DTF \\
\hline Sample Description: & AGR-3/4 DTF Particle Batch \\
\hline Folder containing data: & IIMC-agr\AGR\2-MGEM\R08102201\} $\\
{\hline}$
\end{tabular}

\begin{tabular}{|c|c|c|c|c|c|c|c|}
\hline \multirow{2}{*}{ Particle \# } & \multirow{2}{*}{$\begin{array}{c}\text { Grid } \\
\text { Position }\end{array}$} & \multicolumn{3}{|c|}{ Diattenuation } & \multicolumn{3}{|c|}{ Equivalent $\mathrm{BAFO}=1+3 \mathrm{~N}$} \\
\hline & & Average & St. Dev. & Ave. Error & Average & St. Dev. & Ave. Error \\
\hline 1 & 4,4 & 0.1475 & 0.0174 & 0.0010 & 1.4425 & 0.0522 & 0.0030 \\
\hline 2 & 4,5 & 0.1533 & 0.0127 & 0.0007 & 1.4599 & 0.0381 & 0.0021 \\
\hline 3 & 4,6 & 0.1453 & 0.0192 & 0.0008 & 1.4359 & 0.0576 & 0.0024 \\
\hline 4 & 5,4 & 0.1508 & 0.0129 & 0.0010 & 1.4524 & 0.0387 & 0.0030 \\
\hline 5 & 5,5 & 0.1391 & 0.0156 & 0.0011 & 1.4173 & 0.0468 & 0.0033 \\
\hline 6 & 5,6 & 0.1442 & 0.0196 & 0.0009 & 1.4326 & 0.0588 & 0.0027 \\
\hline 7 & 6,4 & 0.1427 & 0.0131 & 0.0012 & 1.4281 & 0.0393 & 0.0036 \\
\hline 8 & 6,5 & 0.1279 & 0.0118 & 0.0008 & 1.3837 & 0.0354 & 0.0024 \\
\hline 9 & 6,6 & 0.1389 & 0.0187 & 0.0009 & 1.4167 & 0.0561 & 0.0027 \\
\hline 10 & 5,7 & 0.1365 & 0.0169 & 0.0009 & 1.4095 & 0.0507 & 0.0027 \\
\hline \multicolumn{2}{|c|}{ Average } & 0.1426 & 0.0158 & 0.0009 & 1.4279 & 0.0474 & 0.0028 \\
\hline
\end{tabular}

Mean of average BAFo per particle: 1.4279

Standard deviation of average BAFo per particle: 0.0222

\section{Comments}

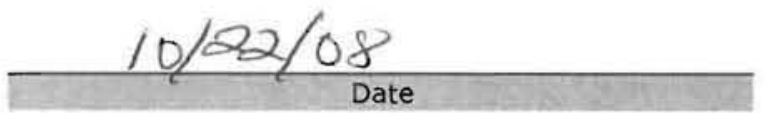


Data Report Form DRF-18A: Measurement of Pyrocarbon Anisotropy using the 2-MGEM - IPyC

\begin{tabular}{|r|l|}
\hline Procedure: & AGR-CHAR-DAM-18 Rev. 1 \\
\hline Operator: & G. E. Jellison \\
\hline Mount ID: & M08101301 \\
\hline Sample ID: & LEU04-02DTF \\
\hline Sample Description: & AGR-3/4 DTF Particle Batch \\
\hline Folder containing data: & \Imc-agr\AGR $\backslash 2$-MGEM\R08102201\} $\\
{\hline}$
\end{tabular}

\begin{tabular}{|c|c|c|c|c|c|c|c|}
\hline \multirow{2}{*}{ Particle \# } & \multirow{2}{*}{$\begin{array}{c}\text { Grid } \\
\text { Position }\end{array}$} & \multicolumn{3}{|c|}{ Diattenuation } & \multicolumn{3}{|c|}{ True BAFo $=(1+\mathrm{N}) /(1-\mathrm{N})$} \\
\hline & & Average & St. Dev. & Ave. Error & Average & St. Dev. & Ave. Error \\
\hline 1 & 4,4 & 0.1475 & 0.0174 & 0.0010 & 1.3460 & 0.0479 & 0.0028 \\
\hline 2 & 4,5 & 0.1533 & 0.0127 & 0.0007 & 1.3621 & 0.0354 & 0.0020 \\
\hline 3 & 4,6 & 0.1453 & 0.0192 & 0.0008 & 1.3400 & 0.0526 & 0.0022 \\
\hline 4 & 5,4 & 0.1508 & 0.0129 & 0.0010 & 1.3552 & 0.0358 & 0.0028 \\
\hline 5 & 5,5 & 0.1391 & 0.0156 & 0.0011 & 1.3232 & 0.0421 & 0.0030 \\
\hline 6 & 5,6 & 0.1442 & 0.0196 & 0.0009 & 1.3370 & 0.0535 & 0.0025 \\
\hline 7 & 6,4 & 0.1427 & 0.0131 & 0.0012 & 1.3329 & 0.0356 & 0.0033 \\
\hline 8 & 6,5 & 0.1279 & 0.0118 & 0.0008 & 1.2933 & 0.0310 & 0.0021 \\
\hline 9 & 6,6 & 0.1389 & 0.0187 & 0.0009 & 1.3226 & 0.0504 & 0.0024 \\
\hline 10 & 5,7 & 0.1365 & 0.0169 & 0.0009 & 1.3162 & 0.0453 & 0.0024 \\
\hline \multicolumn{2}{|c|}{ Average } & 0.1426 & 0.0158 & 0.0009 & 1.3328 & 0.0430 & 0.0025 \\
\hline
\end{tabular}

Mean of average BAFo per particle: 1.3328

Standard deviation of average BAFo per particle: 0.0201

\section{Comments}
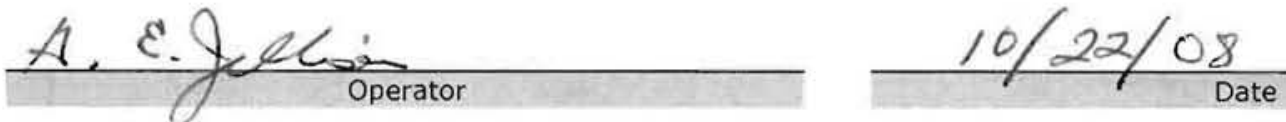


\section{Data Report Form DRF-22: Estimation of Average Particle Weight}

\begin{tabular}{|r|l|}
\hline Procedure: & AGR-CHAR-DAM-22 Rev. 1 \\
\hline Operator: & Dixie Barker \\
\hline Particle Lot ID: & LEU04-02DTF \\
\hline Particle Lot Description: & AGR-3/4 DTF Particle Batch \\
\hline Filename: & \Imc-agr\AGR\ParticleWeight|W08100801_DRF22R1.xIs \\
\hline
\end{tabular}

\begin{tabular}{|r|c|c|c|c|c|}
\hline & Sample 1 & Sample 2 & Sample 3 & Sample 4 & Sample 5 \\
\hline Weight of particles (g): & 0.0701 & 0.0749 & 0.0831 & 0.0862 & 0.0910 \\
\hline Number of particles: & 151 & 160 & 181 & 188 & 197 \\
\hline Average weight/particle (g): & $4.642 \mathrm{E}-04$ & $4.681 \mathrm{E}-04$ & $4.591 \mathrm{E}-04$ & $4.585 \mathrm{E}-04$ & $4.619 \mathrm{E}-04$ \\
\hline
\end{tabular}

Mean average weight/particle (g): $4.624 \mathrm{E}-04$

Standard error in mean average weight/particle $(g): 1.76 \mathrm{E}-06$
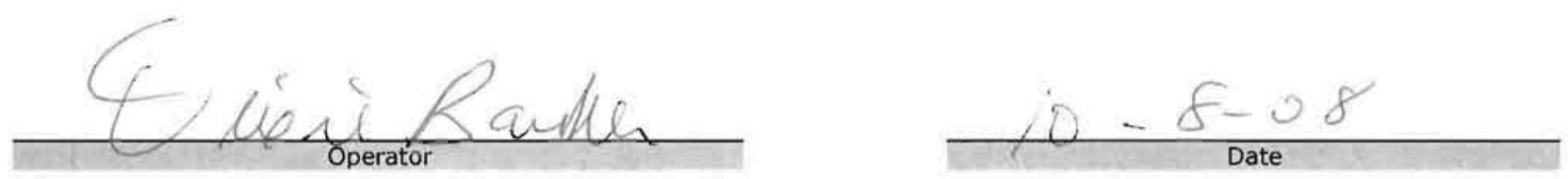
Data Report Form DRF-31: Measurement of Open Porosity using a Mercury Porosimeter

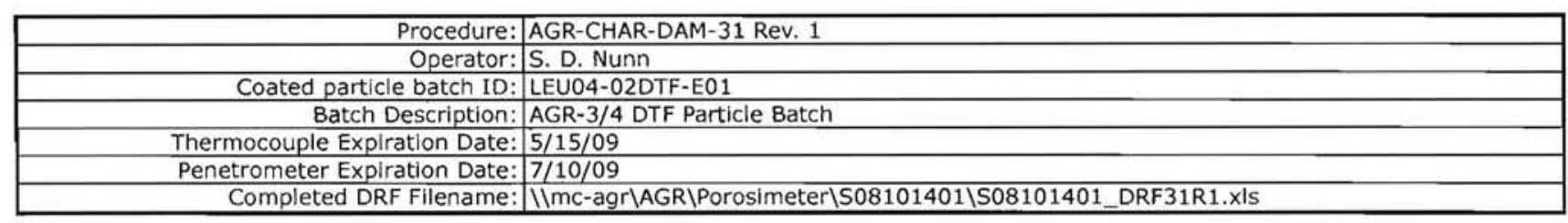

Mean average weight/particle (g): $4.62 \mathrm{E}-04$

Standard error in mean average weight/particle $(g): 1.76 \mathrm{E}-06$

\begin{tabular}{|r|l|}
\hline Weight of particles $(\mathrm{g}):$ & 12.9123 \\
\hline Approximate number of particles: & 27925 \\
\hline Uncertainty in number of particles: & 106 \\
\hline Total envelope volume of sample $(\mathrm{cc}):$ & 1.487 \\
\hline Average envelope volume/particle $(\mathrm{cc}):$ & $5.33 \mathrm{E}-05$ \\
\hline Sample envelope density $(\mathrm{g} / \mathrm{cc}):$ & 8.681 \\
\hline &
\end{tabular}

Average particle diameter (mlcrons): $4.67 \mathrm{E}+02$

Average surface area/particle $(\mathrm{cm} 2): 6.85 \mathrm{E}-03$

Total sample surface area $(\mathrm{cm} 2): 1.91 \mathrm{E}+02$

Intruded mercury volume from $250-10,000 \mathrm{psia}(\mathrm{cc}): 2.38 \mathrm{E}-02$

Open porosity $(\mathrm{ml} / \mathrm{m} 2): 1.24 \mathrm{E}+00$

See note

\section{Comments}

The measured intrusion volume and calculated open porosity was mostly a result of compression of the DTF coating rather than intrusion Into open pores. The actual open porosity is much lower and can not be determined with this method.

S.D. Munn Operator
$1 0 \longdiv { 1 4 } / 0 8$

Date 


\section{For Information Only}

The following sections provide additional information relevant to the LEU04-02DTF particle batch. 


\section{Anomalies observed during inspection by optical microscopy}

Two anomalies were observed during optical microscopy analysis of the designed-to-fail (DTF) particle cross-sections. First, a gap was observed between the kernel and the coating. This had some impact on the image analysis for coating thickness and on the measurement of open porosity. Second, what appears to be low density soot inclusions were observed in the DTF layer.

Figure 1 and Figure 2 show typical cross-sectioned particles from LEU04-02DTF and NUCO425-06DTF batches. A gap between the kernel and DTF layer of 1-2 $\mu \mathrm{m}$ was observed on all the cross-sectioned particles. The image analysis software was not designed to account for the existence of this gap. On a few particles ( $\sim 10 \%$ or less), the inner boundary of the DTF was correctly identified on the pyrocarbon side; on some particles $(\sim 40 \%)$, the boundary was almost completely identified on the kernel side; on the remaining particles $(\sim 50 \%)$, the identified boundary alternated from one side of the gap to the other. The result is that a positive bias was introduced to the average thickness measurement of approximately $1 \mu \mathrm{m}$. This bias does not impact the determination that the coating thickness was within the specified range. In fact, subtracting $1 \mu \mathrm{m}$ to account for this bias brings the average thickness closer to the center of the specified range. Note that this gap was also observed in the GA DTF archive material (Figure 3). Compression of the DTF to close the gap during measurement in the mercury porosimeter made it impossible to determine the open porosity of the layer. This is discussed in the next section.

Figure 1 and Figure 2 also show a second anomaly, which was observed on most of the particles. There is a dark band close to the kernel/coating interface, which indicates an interruption in the pyrocarbon coating. This is probably a layer of lower density carbon "soot", similar to the anomalies sometimes seen in the layers of TRISO coated particles. In most case the layer was only 1-2 $\mu \mathrm{m}$ thick, although a few examples of thicker inclusions were also observed (Figure 4).

The GA archive DTF particles also showed what appear to be low density soot inclusions in the DTF layer, many of them much more severe than those observed in the ORNL particles (Figure 5 and Figure 6). Therefore, it is not surprising that these anomalies, which are related to fluidization problems, were observed in the ORNL particles, which were intentionally coated using conditions close to those use by GA, even though these conditions were determined to not be optimized for particle fluidization during preliminary testing with the ORNL $50 \mathrm{~mm}$ coater. Note that for the analysis of the DTF layer thickness for the GA particles, 8 particles with extremely thick inclusions were not included in the average thickness determination because this would bias the results. 


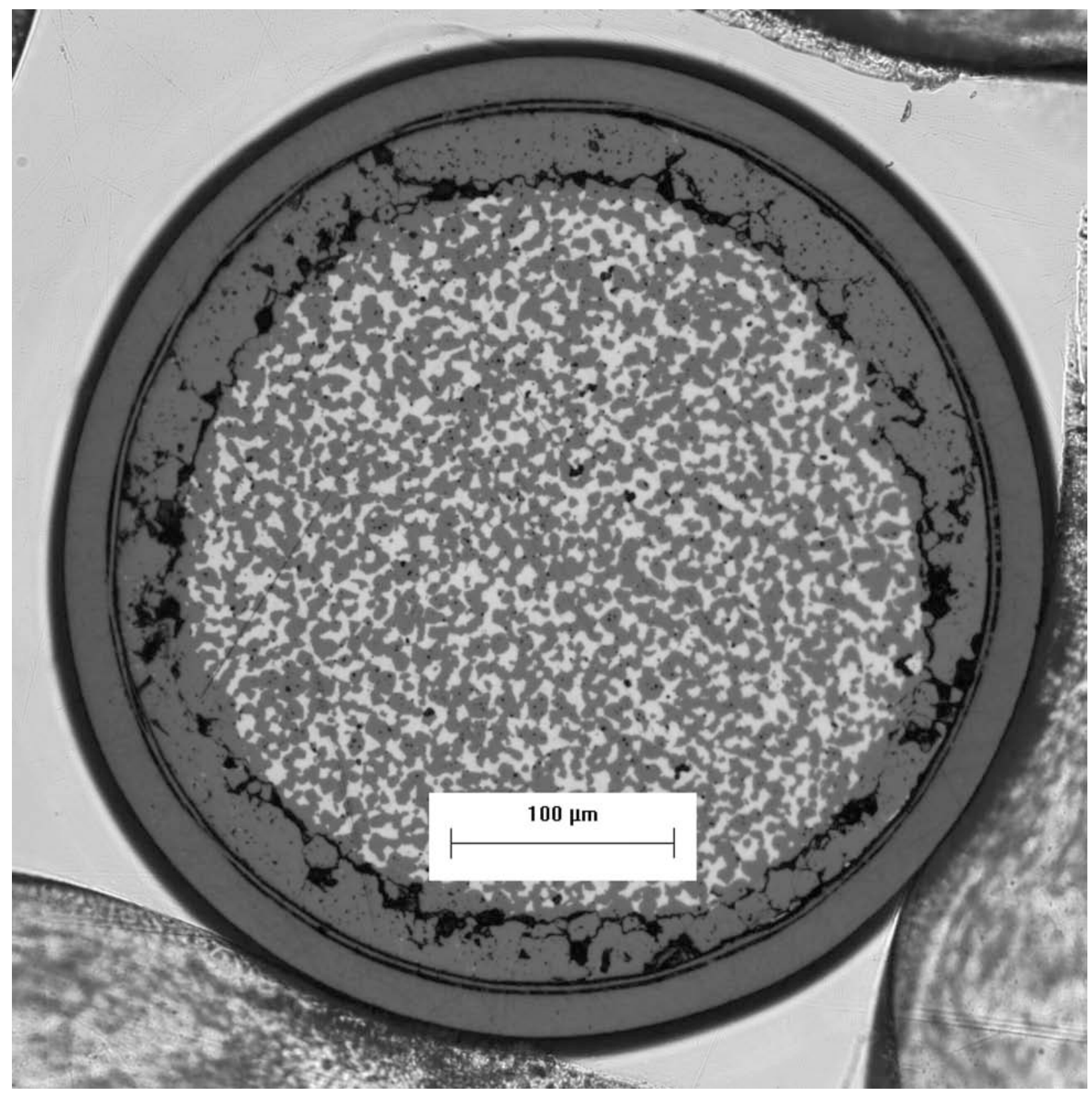

Figure 1. Cross-section of LEU04-02DTF particle. 


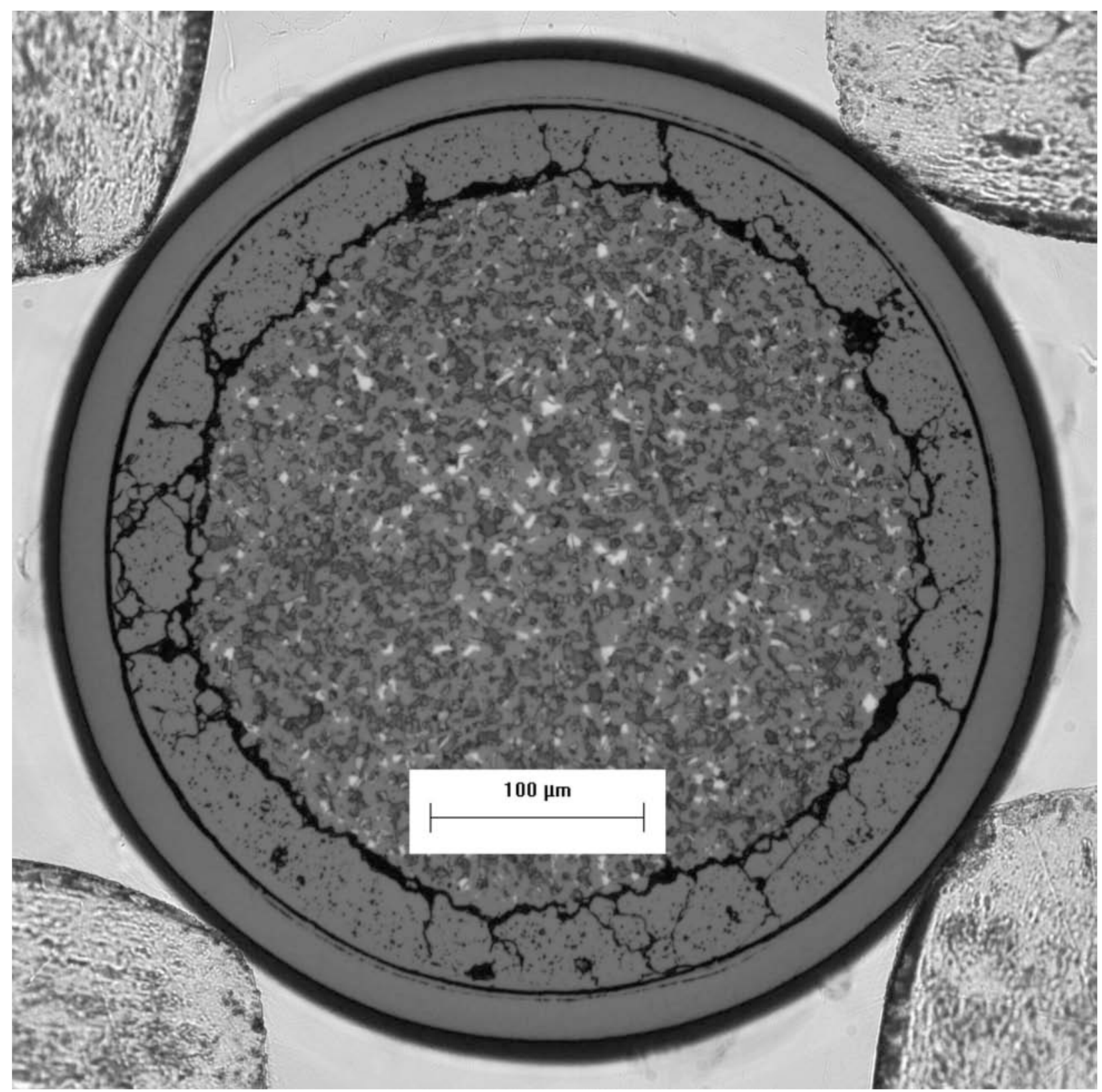

Figure 2. Cross-section of NUCO425-06DTF particle. 


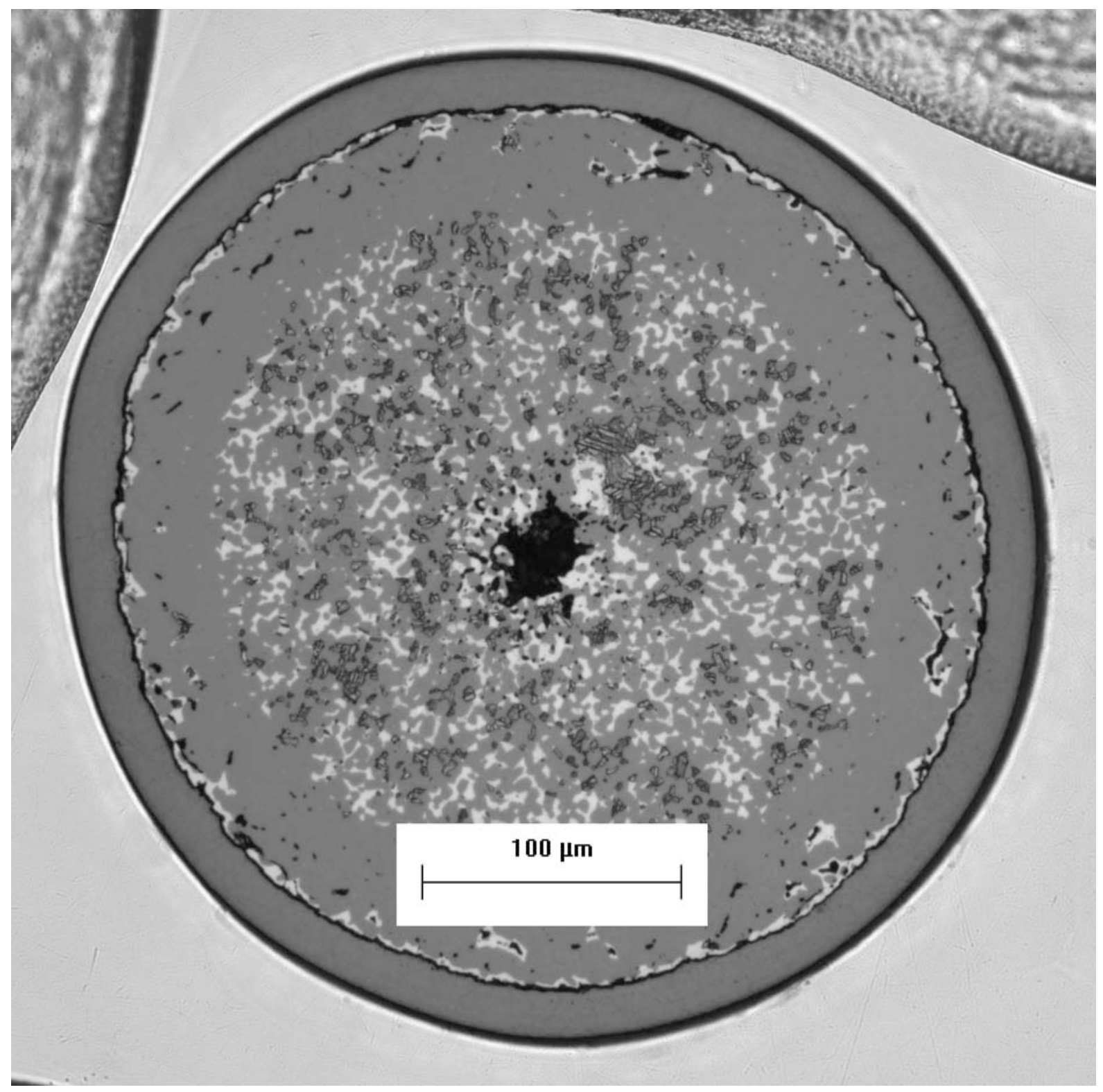

Figure 3. Cross-section of archive GA DTF particle. 


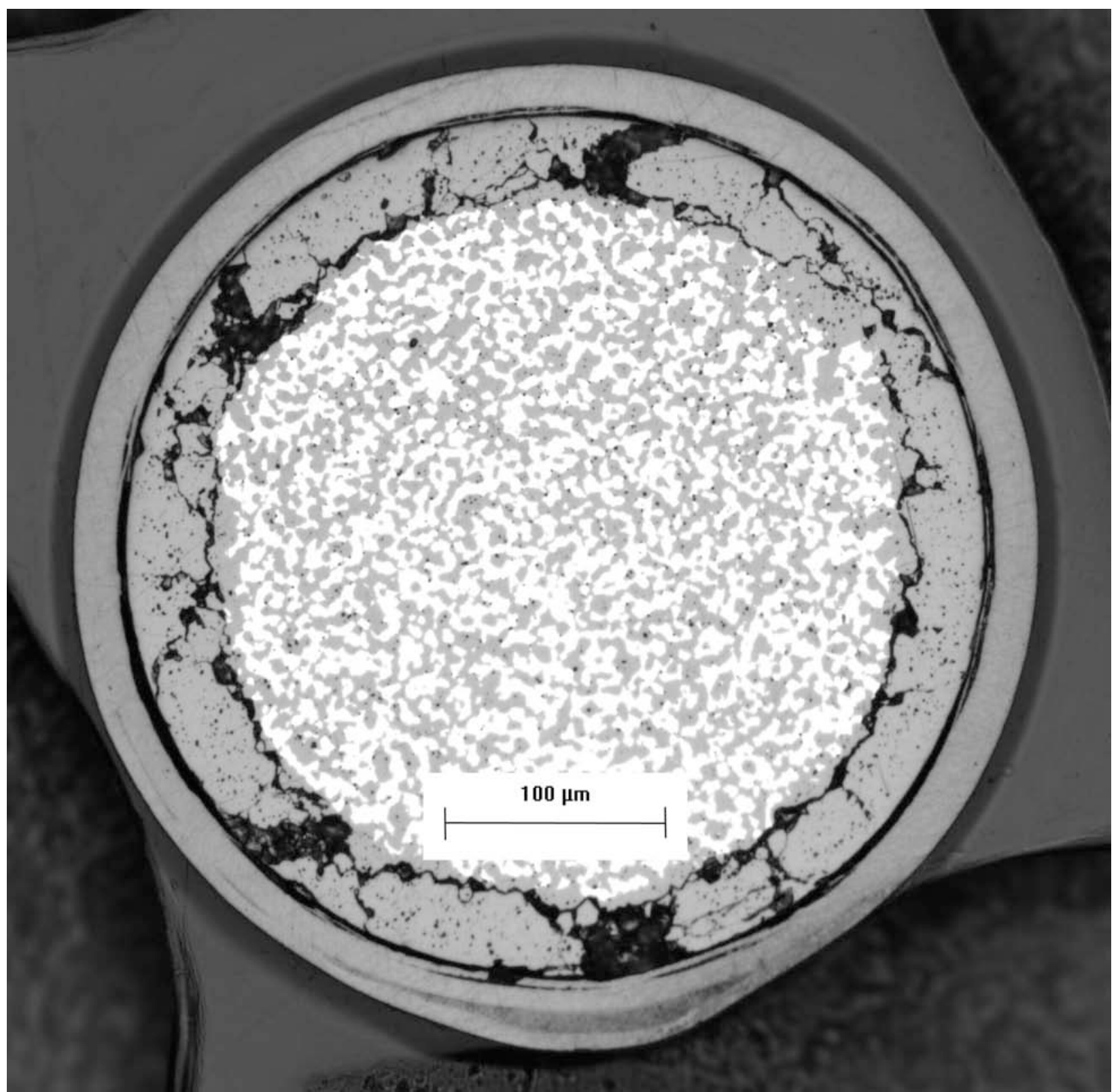

Figure 4. LEU04-02DTF particle cross section showing a thick inclusion in the DTF layer. 


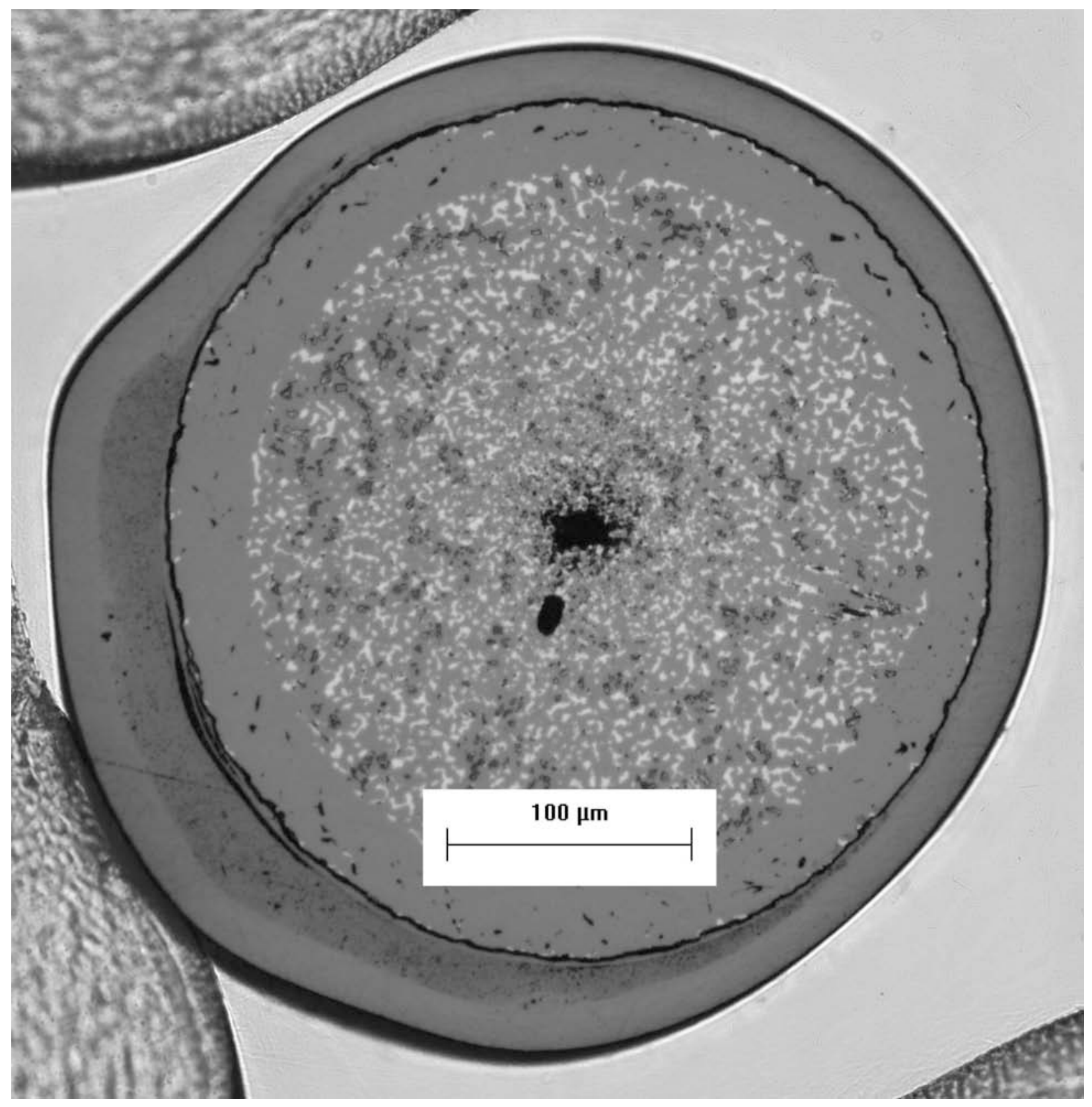

Figure 5. GA DTF with significant inclusion. 


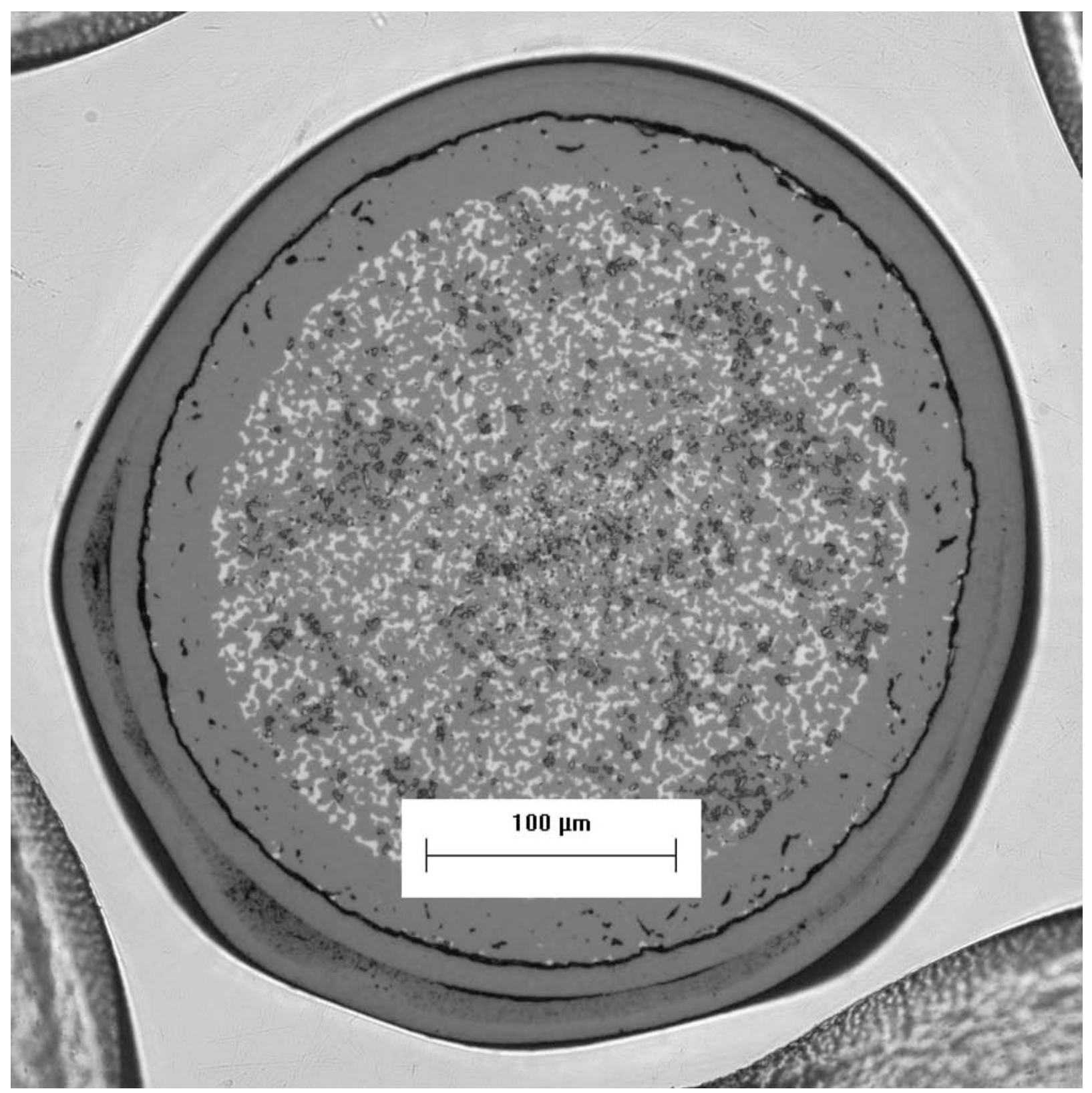

Figure 6. GA DTF with significant inclusion. 


\section{Effect of layer compression on mercury porosimeter measurements}

Two batches of designed-to-fail (DTF) pyrocarbon-coated kernels were evaluated for open porosity using a mercury porosimeter. The particle batches that were examined were NUCO42506DTF and LEU04-02DTF. The kernels used for these two batches were nominally $425 \mu \mathrm{m}$ in diameter and the DTF was nominally $20 \mu \mathrm{m}$ thick. Examination of the intrusion and extrusion curves from the mercury porosimeter indicated that, in a particular pressure range, the particles underwent elastic compression. This elastic compression dominated the volume change and made it impossible to determine the open porosity.

The sample of particles is placed in a penetrometer cell, which is evacuated and back-filled with mercury. Volume reduction as pressure is increased in the penetrometer is interpreted by the porosimeter software as mercury intrusion into the open porosity of a sample. However, in some cases, a portion of this volume reduction may be due to compression of the sample. This effect is observed, for instance, when measuring the open porosity of the inner pyrocarbon, due to compression of the underlying buffer layer. In contrast, compression is negligible when measuring outer pyrocarbon, where the underlying $\mathrm{SiC}$ is not compressible. Elastic compression is indicated by the intruded volume vs. pressure data when a plot of the data shows a linear relationship of intrusion volume versus applied pressure. Elastic compression will also show a recovery in the volume change as pressure is lowered. This extrusion curve should have about the same slope as the intrusion curve in the compression region, with an offset that is related to the actual mercury intrusion or other volume reducing effects such as inelastic compression.

For the NUCO425-06DTF particles, the volume change was approximately linear over a pressure range of about 30 to 8000 psi (Figure 7 and Figure 8). Above 8000 psi, the volume change tapered off, probably due to the cessation of compression for an increasing population of particles in the sample. Above 16000 psi, no more volume change was observed. For the LEU0402DTF particles, the linear compression range was about 150 to 7000 psi (Figure 9 and Figure 10). Again, the volume change tapered off at higher pressure and stopped at about 16000 psi. In both cases, the linear elastic compression occurred in a pressure range that overlapped the pressure span that is used in determining the open porosity, namely 250 to 10,000 psi. Therefore, the open porosity values that would be reported using the standard procedure would be in error because they include both real open porosity and sample compression, the later being the dominant effect. For the NUCO425-06DTF sample, open porosity based on the standard procedure was $1.18 \mathrm{ml} / \mathrm{m}^{2}$, for LEU04-02DTF it was $1.24 \mathrm{ml} / \mathrm{m}^{2}$. However, most of this appears due to compression and the open porosity is believed to be much lower. The dense, shiny surface appearance of the DTF also indicates a low surface porosity.

The particle compression behavior that was observed in the mercury porosimeter can be explained by compression of the DTF against the kernel. Materialographic cross-sections show a narrow gap between the pyrocarbon layer and the kernel (Figure 11 and Figure 12). This gap develops because of a thermal expansion mismatch as the particles are cooled from the coating temperature. It is hypothesized that the coating layer was compressed as pressure was applied in the mercury porosimeter until the gap was closed and the coating came into direct contact with the underlying kernel surface. To corroborate this explanation, calculations were made to see if 
the observed compression volume can be accounted for by the volume of the gap between the coating and the kernel.

The total measured volume reduction, after low pressure filling of the space between the particles in the sample, was about $0.03 \mathrm{~cm}^{3}$ for the NUCO425-06DTF sample and about 0.025 $\mathrm{cm}^{3}$ for the LEU04-02DTF sample. For a kernel diameter of about $425 \mu \mathrm{m}$ and given the number of particles in each sample, this corresponds to a gap between the outer surface of the kernel and the inner surface of the DTF of about $1.8 \mu \mathrm{m}$ for the NUCO425-06DTF sample and about 1.5 $\mu \mathrm{m}$ for the LEU04-02DTF sample. These values are reasonable when compared to the observed gap between the kernel and DTF in the polished cross-sections.

As an additional measurement validation, the particle volume determined using the mercury porosimeter can be compared to results of other measurements. Average measured particle envelope volume was $5.50 \mathrm{E}-5 \mathrm{~cm}^{3}$ for the NUCO425-06DTF sample and $5.33 \mathrm{E}-5 \mathrm{~cm}^{3}$ for the LEU04-02DTF sample. For a NUCO kernel diameter of $421 \mu \mathrm{m}$ (reported by B\&W for lot G73Z-NU-69306), a gap of $1.8 \mu \mathrm{m}$ and a coating thickness of $23.4 \mu \mathrm{m}$ (measured by ORNL), the expected average particle volume is $5.48 \mathrm{E}-5 \mathrm{~cm}^{3}$ for the NUCO425-06DTF sample. For a LEUCO kernel diameter of $426.5 \mu \mathrm{m}$ (reported by B\&W for lot G73I-14-69307), a gap of 1.5 $\mu \mathrm{m}$ and a coating thickness of $20.9 \mu \mathrm{m}$ (measured by ORNL), the expected average particle volume is $5.46 \mathrm{E}-5 \mathrm{~cm}^{3}$ for the LEU04-02DTF sample. Both these values agree within the expected uncertainties of the measurements. 


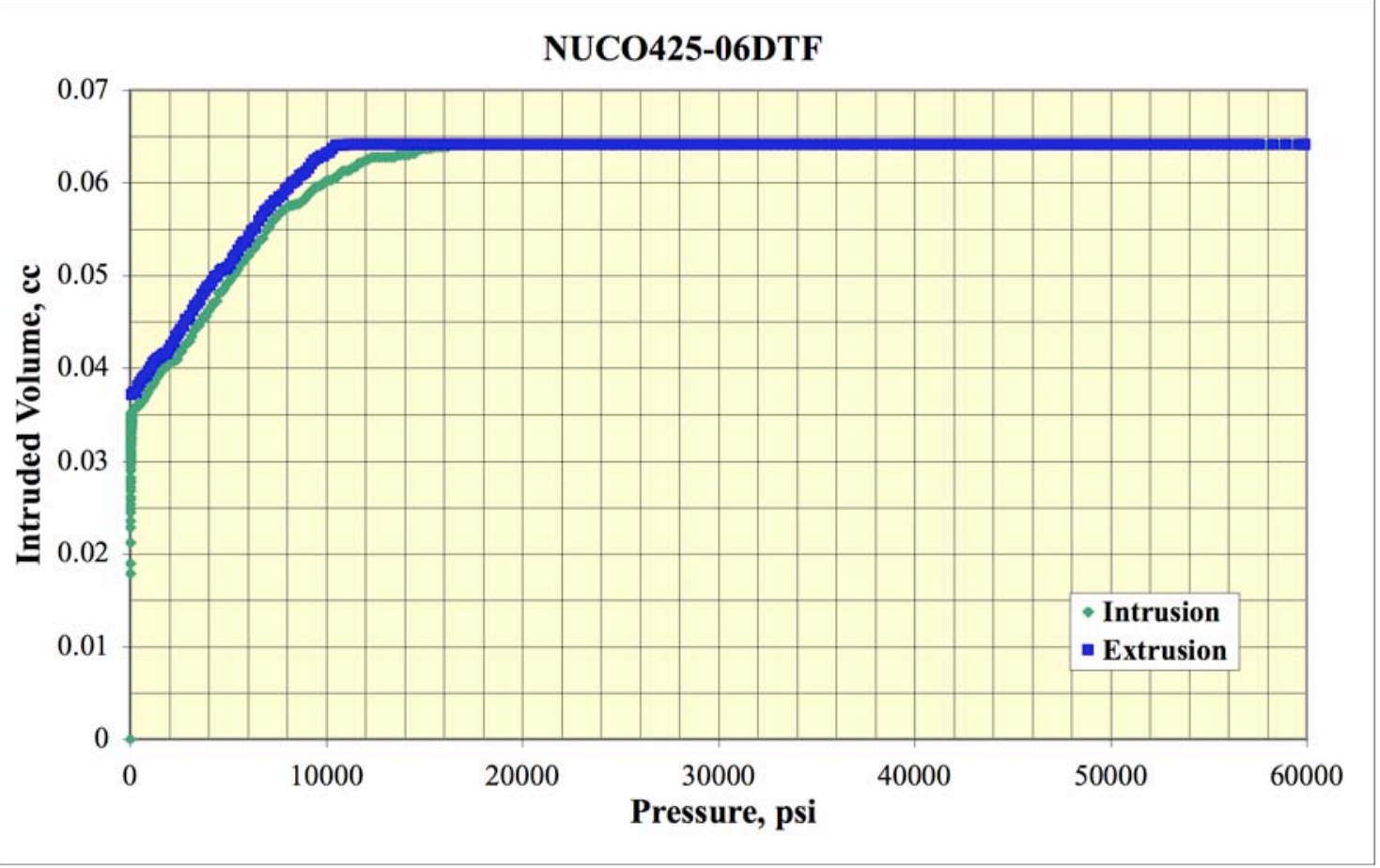

Figure 7. Plot of the mercury porosimeter data for NUCO425-06DTF showing the linear elastic response of the intrusion and extrusion curves over the pressure range of $\sim 30$ to 8000 psi.

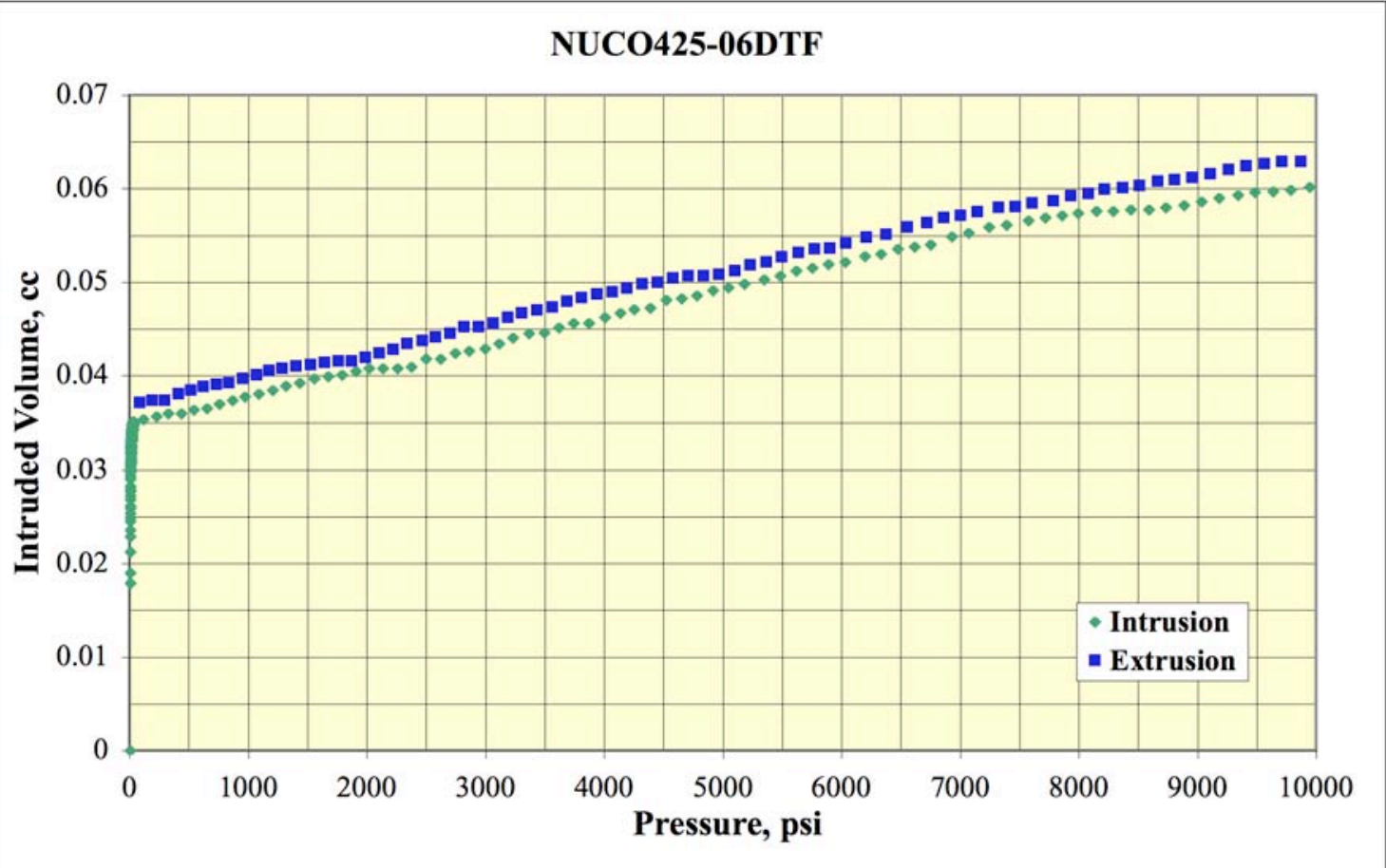

Figure 8. Detail plot of the mercury porosimeter data for NUCO425-06DTF in the linear elastic response range. 


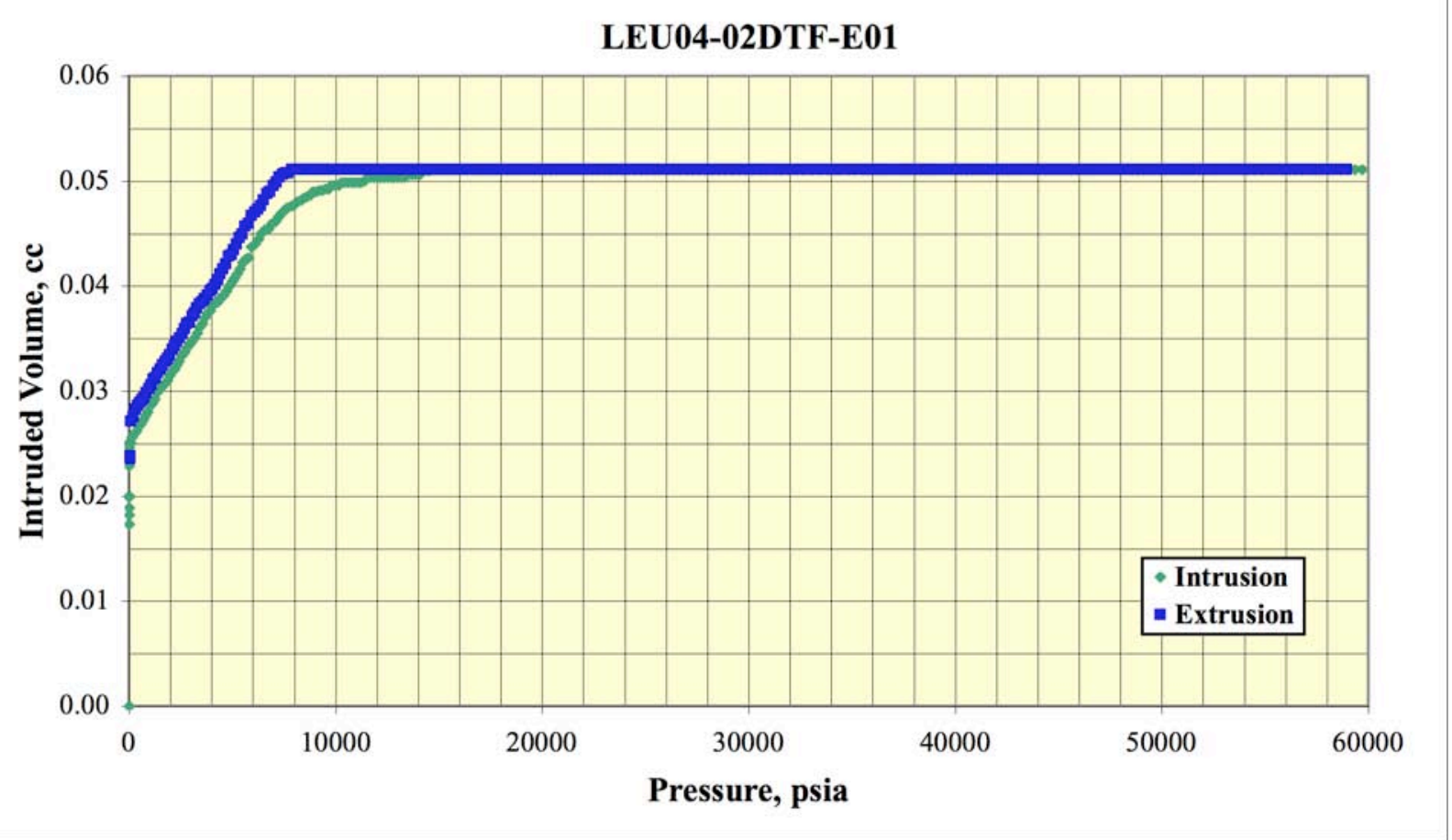

Figure 9. Plot of the mercury porosimeter data for LEU04-02DTF showing the linear elastic response of the intrusion and extrusion curves over the pressure range of $\sim 150$ to 6600 psi.

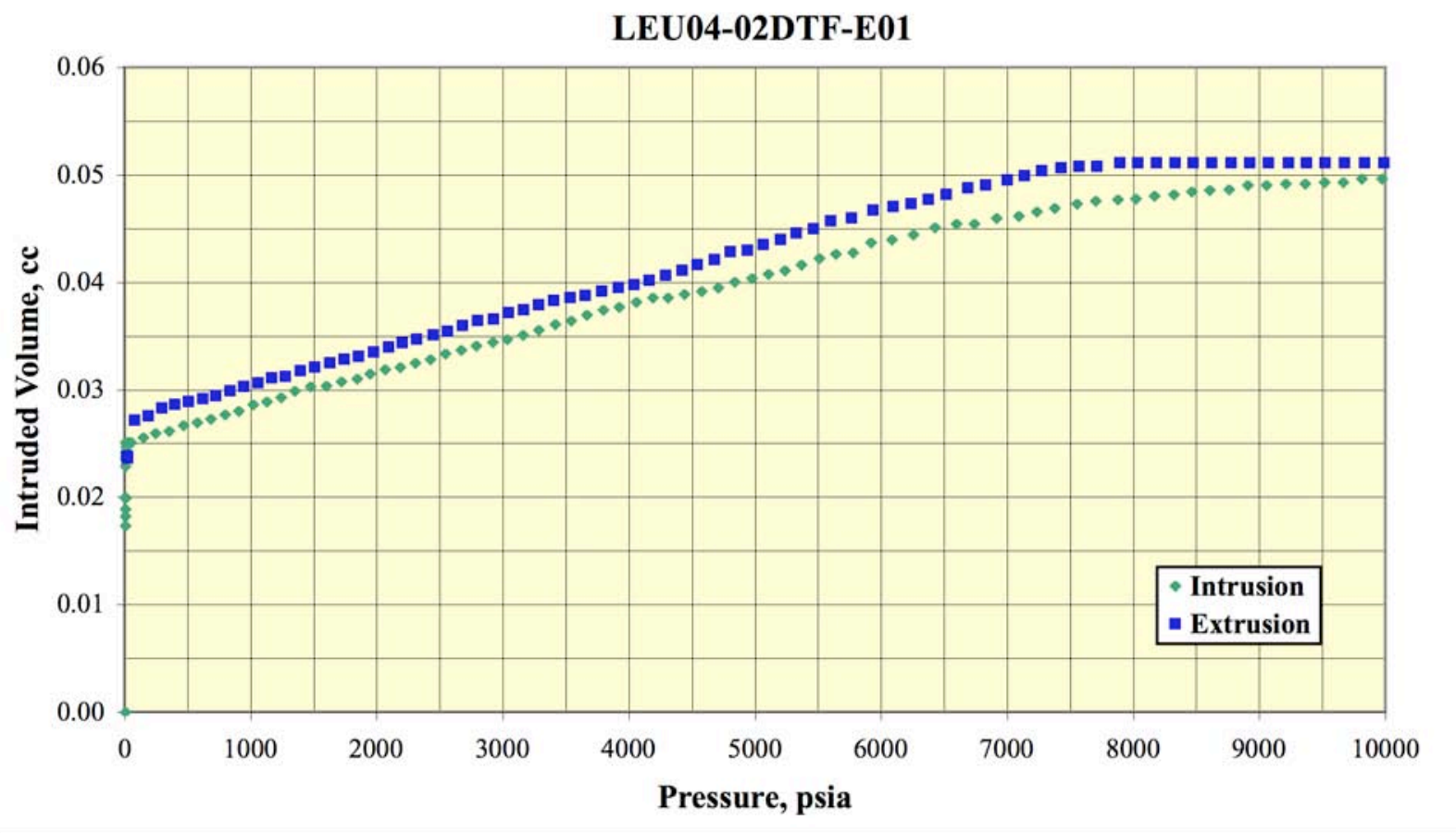

Figure 10. Detail plot of the mercury porosimeter data for LEU04-02DTF in the linear elastic response range. 


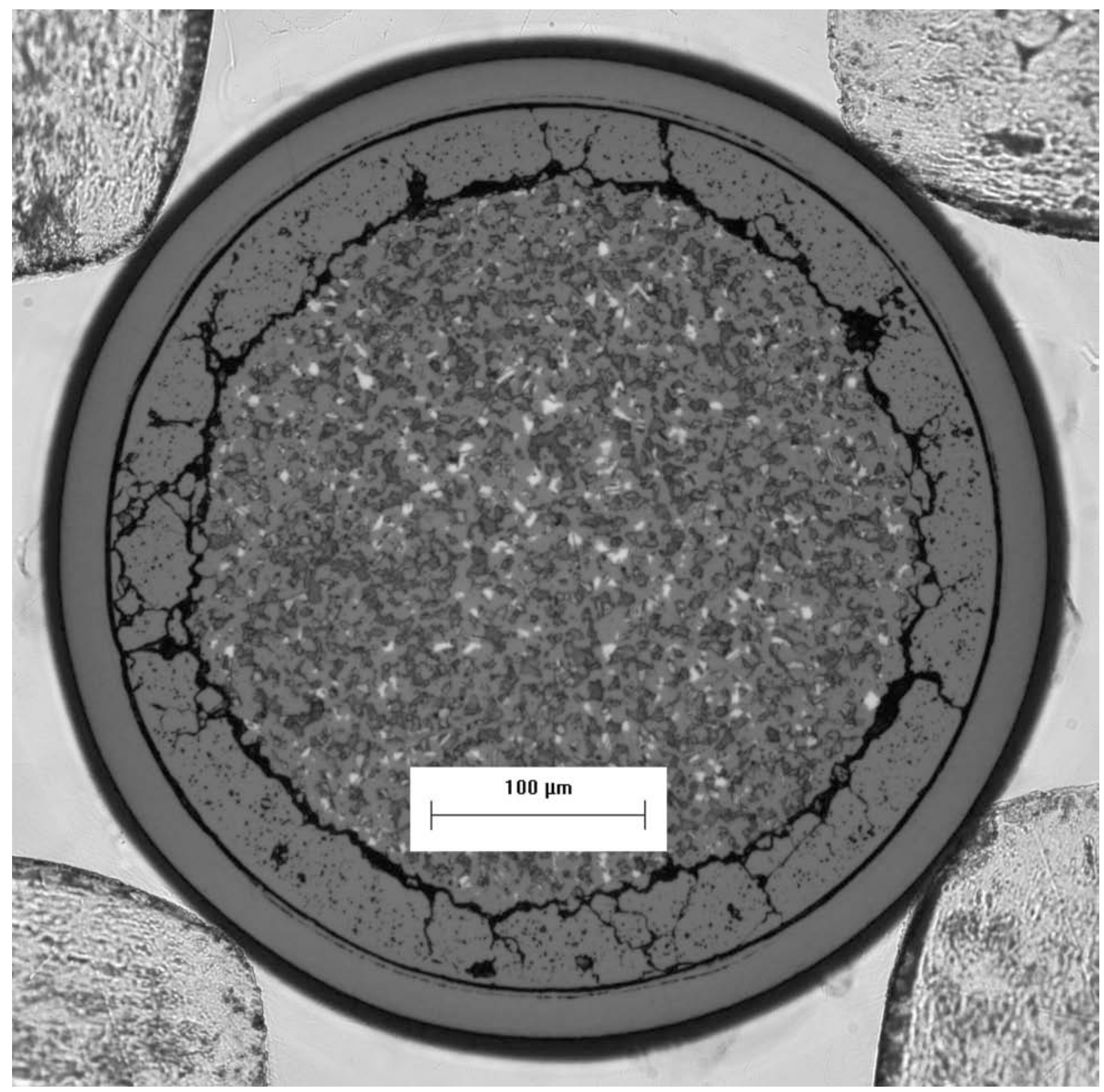

Figure 11. Cross-section of a particle from NUCO425-06DTF. A gap is evident between the kernel and the coating. 


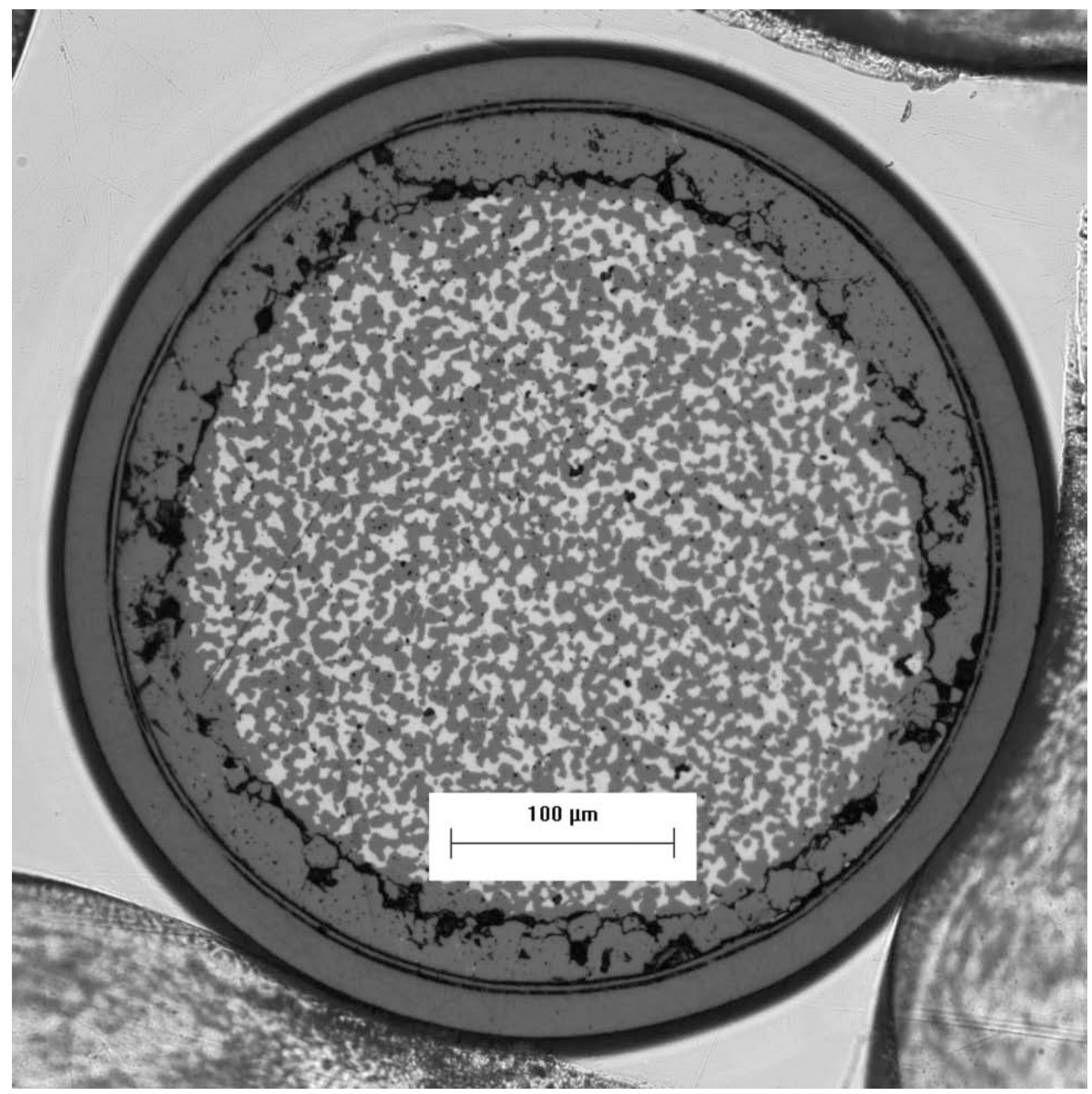

Figure 12. Cross-section of a particle from LEU04-02DTF. A gap is evident between the kernel and the coating. 


\section{Fabrication of NUCO425-06DTF}

ORNL AGR program coating procedure AGR-DTF-COAT-SOP-01 was used to fabricate a DTF coating on natural enrichment UCO kernels using the ORNL Coated Particle Fuel Development Laboratory 50-mm-diameter fluidized bed coater. Table 4 gives a summary of the process conditions and the resulting properties of the DTF coated particles. The coating process conditions met the specifications for DTF particles in section 3.2 of the AGR-3 \& 4 Fuel Product Specification (EDF-6638, Rev.1), with the exception of the coating gas fraction (CGF), for which a deviation request was approved (see note 1 below). A copy of the coating summary sheet from the laboratory log book is included in this section.

Table 4. Summary of DTF Coating Conditions and Results

\begin{tabular}{|c|c|c|}
\hline Parameter & Specified Value & NUCO425-06DTF \\
\hline He $(\mathrm{sccm})$ & Not specified & 12,200 \\
\hline Propylene $(\mathrm{sccm})$ & Not specified & 130 \\
\hline TGF $(\mathrm{sccm})$ & Not specified & 12,330 \\
\hline CGF & $0.011 \pm 0.002$ (note 1$)$ & 0.0105 \\
\hline Coating Temperature $\left({ }^{\circ} \mathrm{C}\right)$ & $1285 \pm 25$ & 1260.7 \\
\hline Coating Time $(\mathrm{min})$. & Not specified & 120 \\
\hline Coating Thickness $(\mu \mathrm{m})$ & $20 \pm 5$ & 23.4 \\
\hline Deposition Rate $(\mu \mathrm{m} / \mathrm{min})$. & $\sim 0.19$ & 0.195 \\
\hline Coating Density $(\mathrm{g} / \mathrm{cc})$ & $1.95 \pm 0.05$ & 1.928 \\
\hline Anisotropy $($ BAFo equiv. $)$ & Not specified & 1.391 \\
\hline
\end{tabular}

Note 1. The coating gas fraction specification in the AGR-3 \& 4 Fuel Product Specification (EDF-6638, Rev.1) was $0.015 \pm 0.0015$. A deviation request (DR-ORNL-AGR-08-01) to operate in the region $0.011 \pm 0.002$ was approved. This region was determined during development efforts to produce coatings of the desired properties. A copy of the approved deviation request is included in this section. 
Surface Processing \& Mechanics Group Materials Science \& Technology Division

Oak Ridge National Laboratory

oak Ridge, Tennessee 37831
AGR-DTF-COAT-SOP-0I ReV. 0

Issue Date 09/15/08

Expire Date $09 / 15 / 11$

Standard Operating Procedure

Fluidized Bed Chemical Vapor Deposition System - DTF

APPENDIX K: COATING SUMMARY

\begin{tabular}{|c|c|c|c|c|}
\hline \multicolumn{2}{|c|}{ Coating Run No. } & \multicolumn{3}{|c|}{$1460425-060 T E$} \\
\hline \multicolumn{2}{|c|}{ Description: } & \multicolumn{3}{|c|}{ DTF particles } \\
\hline \multicolumn{2}{|c|}{ Kernel Lot No. } & \multicolumn{3}{|c|}{ NLCO425-06K } \\
\hline \multicolumn{2}{|c|}{ Kernel Composition } & \multicolumn{3}{|c|}{ NUCO } \\
\hline \multicolumn{2}{|c|}{ Kernel Diameter $(\mu \mathrm{m})$} & \multicolumn{3}{|l|}{420.7} \\
\hline \multicolumn{2}{|c|}{ Kernel Density $\left(\mathrm{g} / \mathrm{cm}^{3}\right)$} & \multicolumn{3}{|l|}{10.97} \\
\hline \multicolumn{2}{|c|}{ Kernel Batch Wt ${ }(\mathrm{g})$} & \multicolumn{3}{|c|}{76.9639} \\
\hline \multicolumn{2}{|c|}{ Kernel surface area $\left(\mathrm{cm}^{2}\right)$} & \multicolumn{3}{|c|}{995.6} \\
\hline \multicolumn{2}{|c|}{ Kernel volume $\left(\mathrm{cm}^{3}\right)$} & \multicolumn{3}{|l|}{11.44} \\
\hline \multicolumn{5}{|c|}{ After Coating } \\
\hline \multicolumn{2}{|c|}{ Coated Particle Batch Wt (g) } & \multicolumn{3}{|c|}{78.9730} \\
\hline \multirow{2}{*}{\multicolumn{2}{|c|}{ Coating Wt. (g) }} & \multicolumn{3}{|c|}{$2.3768 \mathrm{~g}$} \\
\hline & & DTF Paramete & & As-Processed \\
\hline \multicolumn{5}{|c|}{ Carbon } \\
\hline \multicolumn{2}{|c|}{ Coating gases } & \multicolumn{2}{|l|}{$\mathrm{He}+\mathrm{C}_{3} \mathrm{H}_{6}$} & $\mathrm{He}_{2}+\mathrm{C}_{3} \mathrm{H}_{6}$ \\
\hline \multicolumn{2}{|c|}{ TGF } & \multicolumn{2}{|l|}{ Not specified } & 12330 \\
\hline \multicolumn{2}{|l|}{ CGF } & \multicolumn{2}{|l|}{$0.015 \pm 0.0015$} & 0.0105 \\
\hline \multicolumn{2}{|c|}{ CGR } & \multicolumn{2}{|c|}{1 (Propylene only) } & 1 \\
\hline Temperatur & & $1285 \pm 25^{\circ} \mathrm{C}$ & & 1260.7 \\
\hline Helium flow & rate $\left(\mathrm{cm}^{3} / \mathrm{min}\right)$ & Not specified & & 12200 \\
\hline Propylene fl & W rate $\left(\mathrm{cm}^{3} / \mathrm{min}\right)$ & Not specified & & 130 \\
\hline Time & & Not specified & & $120 \min$ \\
\hline Coating rate & $(\mu \mathrm{m} / \mathrm{min})$ & $\sim 0.19$ & & 0.195 \\
\hline Coating thic & ness $(\mu \mathrm{m})$ & $20 \pm 5$ & & 234 \\
\hline Coating Der & sity $\left(\mathrm{g} / \mathrm{cm}^{3}\right)$ & $1.95 \pm 0.05$ & & 1.9279 \\
\hline Comments/ & Jotes: & & & \\
\hline Operator: & $A \cos 20$ & 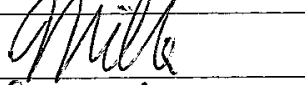 & Date: & $9-26-08$ \\
\hline Verified by: & Do & $\operatorname{lal}$ & Date: & $10 / 27 / 08$ \\
\hline QAS: & & & Date: & $10 / 27 / 08$ \\
\hline
\end{tabular}




\begin{tabular}{|c|c|c|}
\hline \multicolumn{2}{|l|}{$\begin{array}{l}\text { 1. DEVIATION NO: } \\
\text { DR- ORNL-AGR-08-01 }\end{array}$} & 2. DIVISIO:V: Materials Science\&Tech. \\
\hline \multicolumn{2}{|l|}{$\begin{array}{l}\text { 3. PROJECT TITLE: } \\
\text { N/A }\end{array}$} & $\begin{array}{l}\text { 4. PROGRAM: } \\
\text { Advanced Gas Reactor Fuel Developritit ! and Qualification }\end{array}$ \\
\hline $\begin{array}{l}\text { 5. ITEM/ACTIVITY NAME: } \\
\text { DTF Coating for AGR-3/4 }\end{array}$ & & $\begin{array}{l}\text { 6. SPECIFICATION/PROCEDURE: } \\
\text { AGR Program/NL Specilication \#EDF.66: } 38 \text {, Revision } 1\end{array}$ \\
\hline $\begin{array}{l}\text { 7. DRAWING NO: } \\
\text { N/A }\end{array}$ & $\begin{array}{l}\text { 8. SHOP ORDER: } \\
\text { N/A }\end{array}$ & $\begin{array}{l}\text { 9. WORK/PU]:LCHASE: } \\
\text { N/A }\end{array}$ \\
\hline \multicolumn{3}{|c|}{ 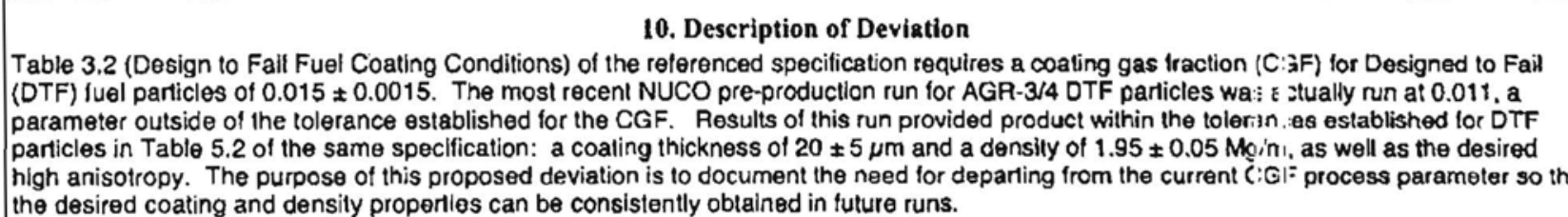 } \\
\hline
\end{tabular}

the desired coating and density properlies can be consistently oblained in future runs.

\section{Justification and Limitations}

Though the CGF of NUCO425-05DTF did not fall within the process condition tolerance provided in Table 3.2 , thi) ( 2 ating process is reproducible and has demonstrated the ability to produce particles with the desired coating thickness and density e: tablished in Table 5.2 . Further coating development in an attempt to adhere to process conditions within the specification that produce far icles thal meet the product specification is not thought to be feasible, and is therefore not recommended.

It is recommended that the CGF process parameter of $0.011 \pm 0.002$ be used in the planned NUCO verilication rut1, : nd in subsequent runs to produce the LEUCO DTF particles.

12. Requested by: J. H. Miller

13. Title: Change to DTF Coating Gas Fraction

15. Drawing/Document is to be revised $\square$ NO $\square$ YES (If yes, list):

\begin{tabular}{|l|l|l|l|l|}
\hline Approved by: & $\begin{array}{l}\text { 16. Task Leader: } \\
\text { J. D. Hunn }\end{array}$ & Date: \\
\cline { 2 - 5 } As Appropriatc & $\begin{array}{l}\text { 17. Requirement Originator: } \\
\text { INL Rep. }\end{array}$ & $\begin{array}{l}\text { Date: } \\
9-22-08\end{array}$ & $\begin{array}{l}\text { 19. Item User: } \\
\text { N/A }\end{array}$ \\
\cline { 2 - 5 } & $\begin{array}{l}\text { 18. QA Group } \\
\text { M. C. Vance }\end{array}$ & $\begin{array}{l}\text { 20. Other: } \\
\text { N/A }\end{array}$ & Date: \\
\hline
\end{tabular}

ORNI_313/Page I of 3 (4-2003) 


\section{QC Acceptance Test of NUCO425-06DTF}

Immediately prior to fabrication of the LEU04-02DTF particle batch on the low enrichment UCO kernels, NUCO425-06DTF was fabricated with natural enrichment UCO kernels using identical conditions, in order to qualify that the process would produce a product that would likely meet the specification. The full AGR-CHAR-PIP-08 product inspection plan was used to characterize the NUCO425-06DTF particle batch. This section contains the inspection report form (IRF-08) and associated data report forms resulting from that analysis. The NUCO42506DTF particle batch was found to meet the specifications for DTF particles in section 5 of the AGR-3 \& 4 Fuel Product Specification (EDF-6638, Rev.1).

Open porosity, which was specified for measurement only, could not be determined due to compression of the DTF coating. This is noted in DRF-31 and described in detail in the section "Effect of layer compression on mercury porosimeter measurements". 
ORNL/TM-2008/193

Inspection Revert Form IRF-08: AGR-3/4 Designed to Fall Coated Particle Batches

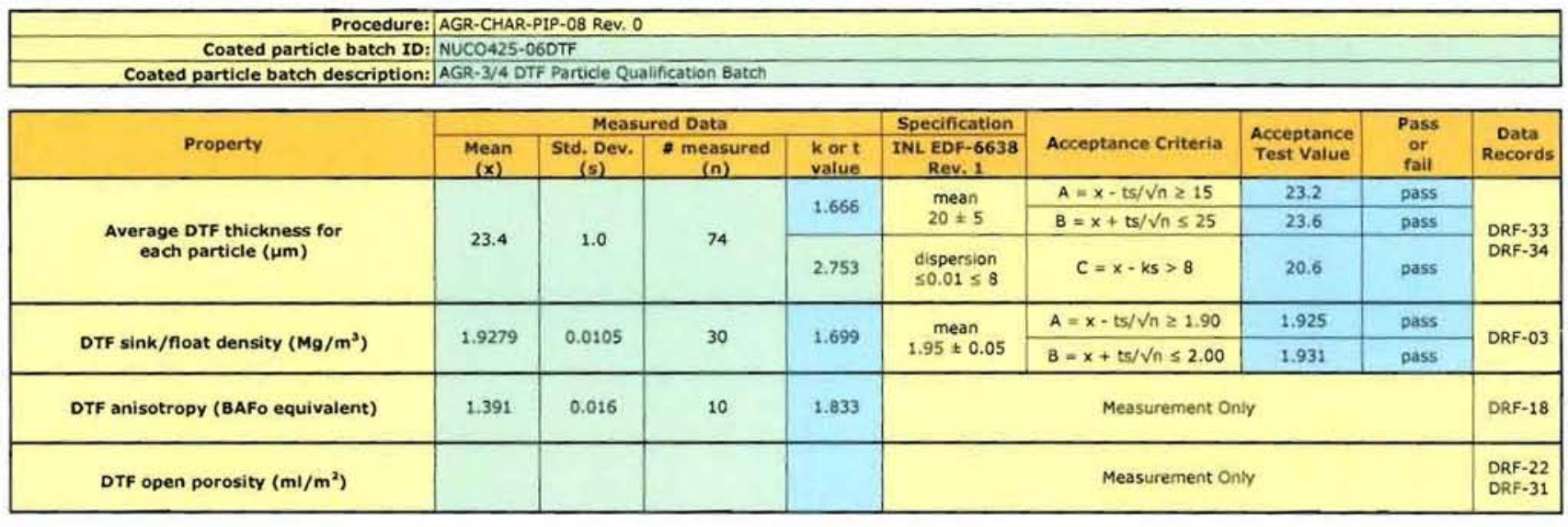
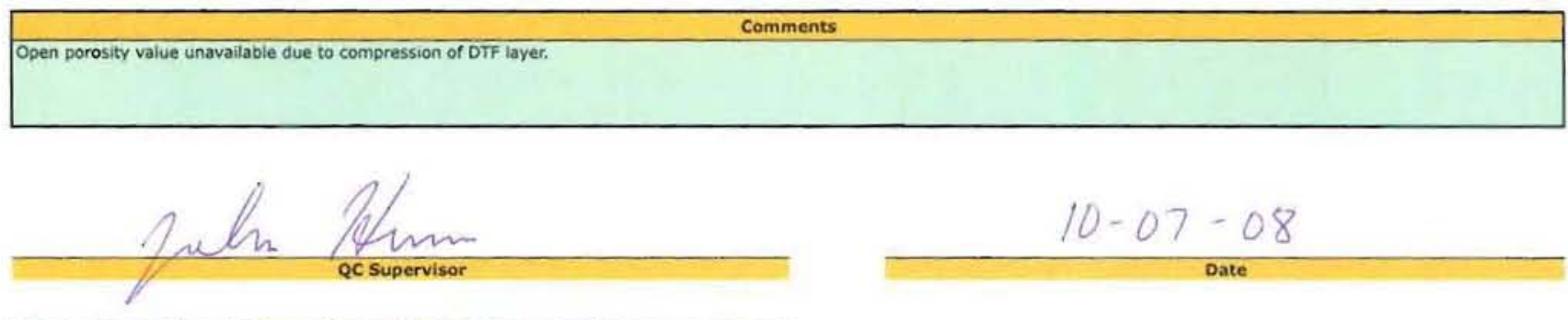

$$
10-07-08
$$

Accept coated particle batch (Yes or $\mathrm{No}$ ):

Yes

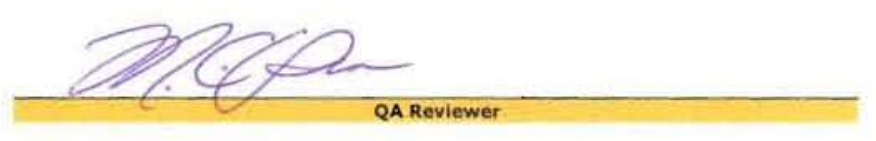

45 


\section{Data Report Form DRF-33: Imaging of Small Particle Cross-sections Usine an Optical Microscope System}

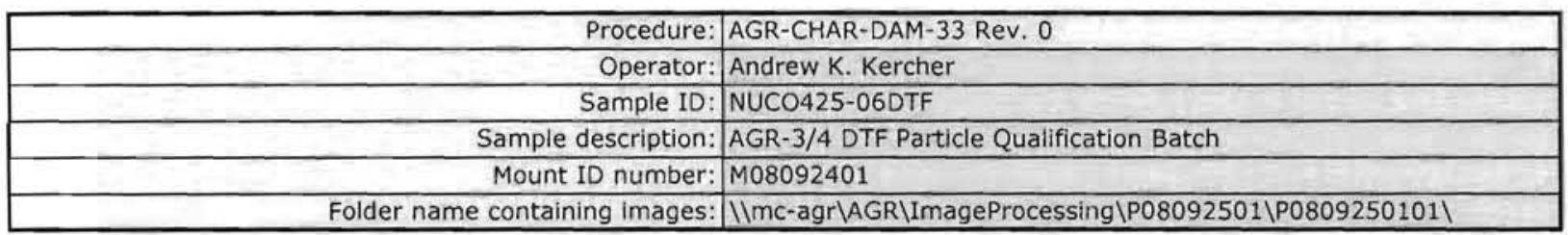

\begin{tabular}{|r|r|}
\hline DMR calibration expiration date: & $9 / 15 / 2009$ \\
\hline Calibrated pixels/micron: & 4.4833 \\
\hline Stage micrometer calibration expiration date: & $2 / 13 / 2009$ \\
\hline Measured value for $300 \mu \mathrm{m}$ in stage micrometer image $(\mu \mathrm{m}):$ & 299.8 \\
\hline
\end{tabular}

\begin{tabular}{|c|c|c|c|}
\hline \multicolumn{4}{|c|}{ Polish-down distance $\mathrm{n}, \mathrm{m}(\mu \mathrm{m})$} \\
\hline 2,2 & 2,8 & 8,2 & 8,8 \\
\hline \hline 185 & 183 & 180 & 171 \\
\hline
\end{tabular}

\begin{tabular}{|c|c|c|c|c|}
\hline \multicolumn{5}{|c|}{ Approximate layer width in polish plane $(\mu \mathrm{m})$} \\
\hline Kernel radius & Layer 1 & Layer 2 & Layer 3 & Layer 4 \\
\hline 206 & 23 & & & \\
\hline
\end{tabular}
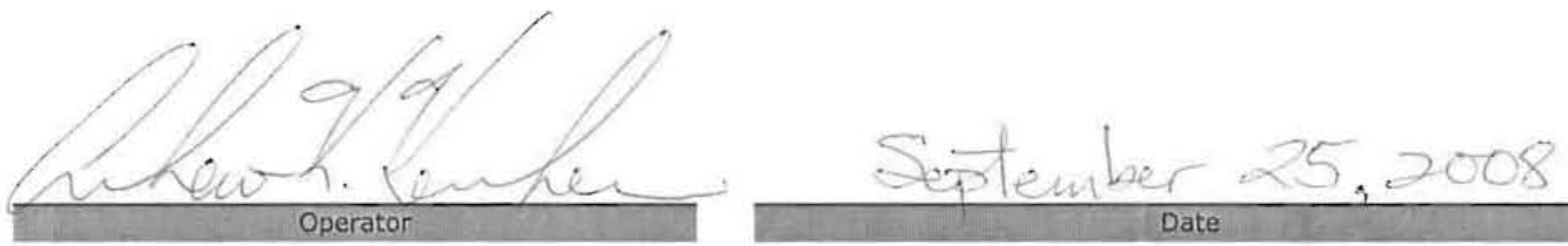


\begin{tabular}{|c|c|}
\hline Procedure: & AGR-CHAR-DAM-34 Rev. 0 \\
\hline Operator: & Andrew K. Kercher \\
\hline Folder name containing images: & 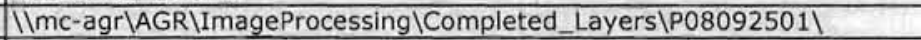 \\
\hline Sample ID: & NUCO $425-06 \mathrm{DTF}$ \\
\hline Sample Description: & AGR-3/4 DTF Particle Qualification Batch \\
\hline Folder name containing processed data: & 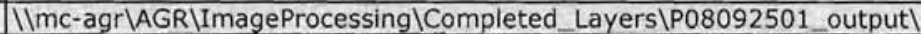 \\
\hline
\end{tabular}

\begin{tabular}{|r|r|}
\hline Number of layers analyzed: & 74 \\
\hline Mean of the average layer 1 thickness of each particle $(\mu \mathrm{m}):$ & 23.4 \\
\hline Standard deviation in the average layer 1 thickness of each particle $(\mu \mathrm{m}):$ & 1.0 \\
\hline
\end{tabular}

\section{Distribution of the average layer 1 thickness (top binned)}

\begin{tabular}{|c|c|}
\hline Layer 1 Thickness $(\mu \mathrm{m})$ & Frequency \\
\hline \hline$<12$ & 0 \\
\hline 13 & 0 \\
\hline 14 & 0 \\
\hline 15 & 0 \\
\hline 16 & 0 \\
\hline 17 & 0 \\
\hline 18 & 0 \\
\hline 19 & 0 \\
\hline 20 & 0 \\
\hline 21 & 1 \\
\hline 22 & 7 \\
\hline 23 & 15 \\
\hline 24 & 28 \\
\hline 25 & 20 \\
\hline 26 & 3 \\
\hline$>26$ & 0 \\
\hline & \\
\hline
\end{tabular}
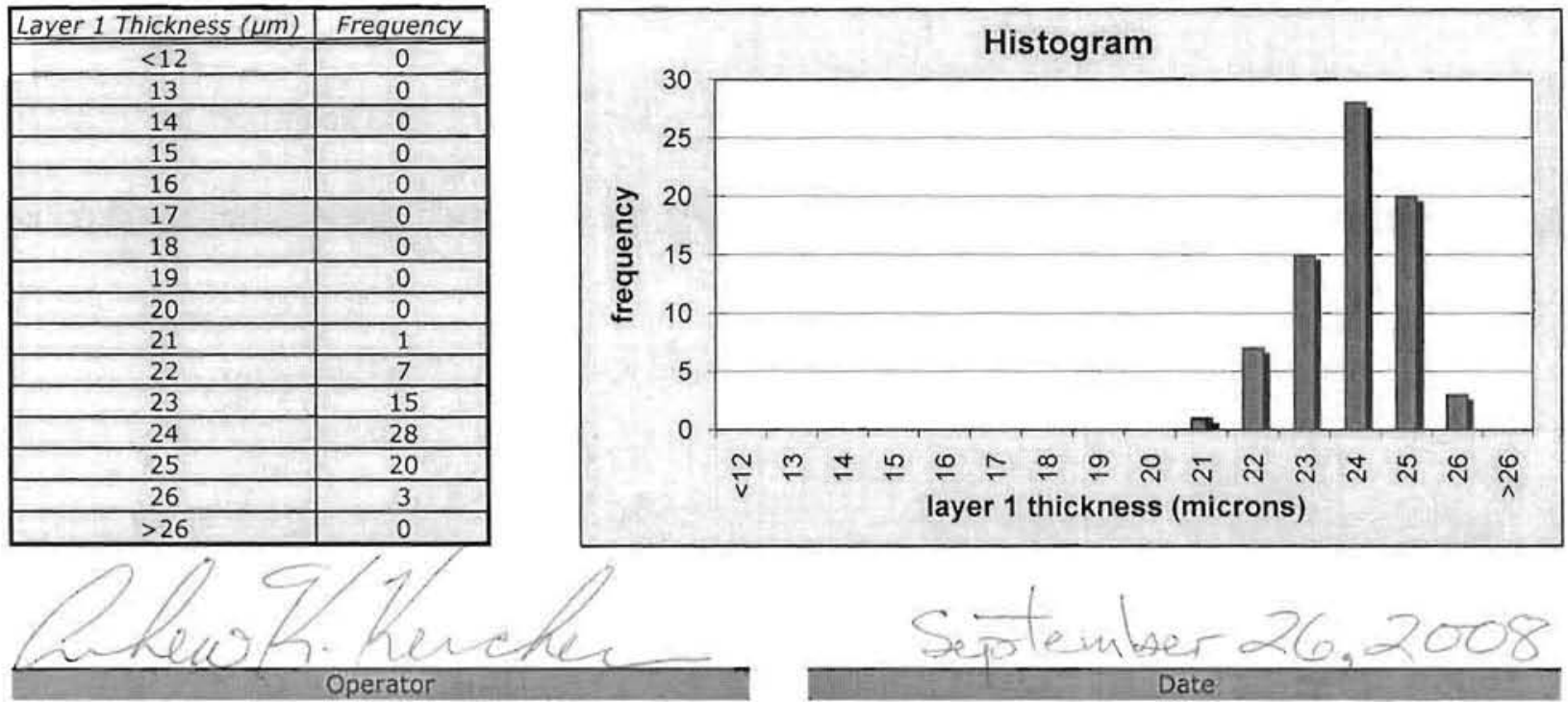
Data Report Form DRF-03: Measurement of PyC Density using a Density Gradient Column

\begin{tabular}{|r|l|}
\hline Procedure: & AGR-CHAR-DAM-03 Rev. 3 \\
\hline Operator: & Dixie Barker \\
\hline Filename: & IImC-agrVAGRIDensityColumnJD08092501_DRF03R3.xis \\
\hline Sample ID: & NUCO425-06DTF-C01 \\
\hline Sample description: & AGR-3/4 DTF Particie Quallfication Batch \\
\hline Float expiration date: & $07 / 2012$ \\
\hline Gauge expiration date: & $11 / 2008$ \\
\hline Bath temperature: & $22.8^{\circ} \mathrm{C}$ \\
\hline
\end{tabular}

\begin{tabular}{|c|c|c|c|}
\hline \multicolumn{4}{|c|}{ Calibrated Floats } \\
\hline Density & $\begin{array}{l}\text { Top of } \\
\text { Float }\end{array}$ & $\begin{array}{c}\text { Bottom of } \\
\text { Float }\end{array}$ & $\begin{array}{c}\text { Center of } \\
\text { Mass }\end{array}$ \\
\hline 1.800 & 127.76 & 137.28 & 132.52 \\
\hline 1.850 & 240.53 & 248.99 & 244.76 \\
\hline 1.900 & 351.02 & 356.65 & 353.84 \\
\hline 1.950 & 455.45 & 462.77 & 459.11 \\
\hline 2.000 & 553.69 & 560.79 & 557.24 \\
\hline
\end{tabular}

\begin{tabular}{|c|c|c|c|}
\hline \multicolumn{4}{|c|}{ Linear Fit } \\
\hline slope & StDev & intercept & StDev \\
\hline \hline $4.70 \mathrm{E}-04$ & $2.85 \mathrm{E}-06$ & $1.74 \mathrm{E}+00$ & $1.07 \mathrm{E}-03$ \\
\hline
\end{tabular}

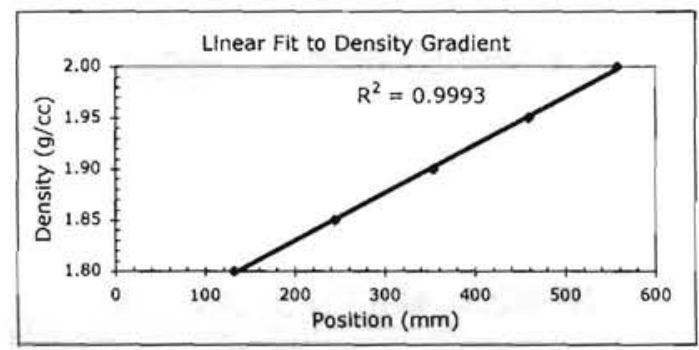

\begin{tabular}{|c|c|c|c|c|c|c|c|c|}
\hline \multicolumn{9}{|c|}{ Sample Density } \\
\hline $\begin{array}{l}\text { Fragment } \\
\text { Number }\end{array}$ & $\begin{array}{l}\text { Fragment } \\
\text { Position }\end{array}$ & $\begin{array}{l}\text { Calculated } \\
\text { Density }\end{array}$ & $\begin{array}{l}\text { Fragment } \\
\text { Number }\end{array}$ & $\begin{array}{l}\text { Fragment } \\
\text { Position }\end{array}$ & $\begin{array}{c}\text { Calculated } \\
\text { Density }\end{array}$ & $\begin{array}{l}\text { Fragment } \\
\text { Number }\end{array}$ & $\begin{array}{l}\text { Fragment } \\
\text { Position }\end{array}$ & $\begin{array}{l}\text { Calculated } \\
\text { Density }\end{array}$ \\
\hline 1 & 386.72 & 1.9175 & 26 & 422.23 & 1.9342 & 51 & & \\
\hline 2 & 388.02 & 1.9181 & 27 & 428.75 & 1.9372 & 52 & & \\
\hline 3 & 391.39 & 1.9197 & 28 & 455.78 & 1.9499 & 53 & & \\
\hline 4 & 392.94 & 1.9204 & 29 & 478.08 & 1.9604 & 54 & & \\
\hline 5 & 393.43 & 1.9206 & 30 & 468.56 & 1.9559 & 55 & & \\
\hline 6 & 394.92 & 1.9213 & 31 & & & 56 & & \\
\hline 7 & 394.99 & 1.9214 & 32 & & & 57 & & \\
\hline 8 & 395.85 & 1.9218 & 33 & & & 58 & & \\
\hline 9 & 396.15 & 1.9219 & 34 & & & 59 & & \\
\hline 10 & 394.94 & 1.9213 & 35 & & & 60 & & \\
\hline 11 & 396.76 & 1.9222 & 36 & & & 61 & & \\
\hline 12 & 397.61 & 1.9226 & 37 & & & 62 & & \\
\hline 13 & 399.91 & 1.9237 & 38 & & & 63 & & \\
\hline 14 & 399.91 & 1.9237 & 39 & & & 64 & & \\
\hline 15 & 401.11 & 1.9242 & 40 & & & 65 & & \\
\hline 16 & 402.43 & 1.9249 & 41 & & & 66 & & \\
\hline 17 & 403.88 & 1.9255 & 42 & & & 67 & & \\
\hline 18 & 404.33 & 1.9258 & 43 & & & 68 & & \\
\hline 19 & 407.29 & 1.9271 & 44 & & & 69 & & \\
\hline 20 & 408.27 & 1.9276 & 45 & & & 70 & & \\
\hline 21 & 408.81 & 1.9279 & 46 & & & 71 & & \\
\hline 22 & 409.67 & 1.9283 & 47 & & & 72 & 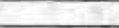 & \\
\hline 23 & 411.79 & 1.9293 & 48 & & & 73 & & \\
\hline 24. & 412.06 & 1.9294 & 49 & & & 74 & & \\
\hline 25 & 417.55 & 1.9320 & 50 & & & 75 & & \\
\hline \multicolumn{4}{|c|}{ Average density of PyC fragments: } & \multicolumn{5}{|c|}{1.9279} \\
\hline \multirow{2}{*}{\multicolumn{4}{|c|}{$\begin{array}{l}\text { Standard deviation in density of PyC fragments: } \\
\text { Uncertainty in calculated density of PyC fragments: }\end{array}$}} & \multicolumn{5}{|c|}{0.0105} \\
\hline & & & & \multicolumn{5}{|c|}{0.0017} \\
\hline
\end{tabular}

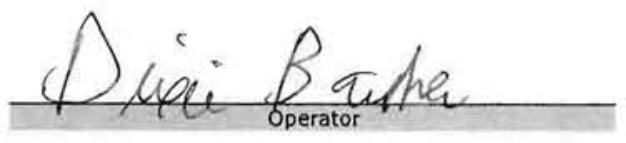

$$
9-25-08
$$


Data Report Form DRF-18A: Measurement of Pyrocarbon Anisotropy using the 2-MGEM - IPyC

\begin{tabular}{|r|l|}
\hline Procedure: & AGR-CHAR-DAM-18 Rev. 1 \\
\hline Operator: & G. E. Jellison \\
\hline Mount ID: & M08092401 \\
\hline Sample ID: & NUCO425-06DTF \\
\hline Sample Description: & AGR-3/4 DTF Particle Qualification Batch \\
\hline Folder containing data: & $\backslash \backslash \mathrm{MC}$-agr\AGR $\backslash 2-M G E M \backslash R 08100201 \backslash$ \\
\hline
\end{tabular}

\begin{tabular}{|c|c|c|c|c|c|c|c|}
\hline \multirow{2}{*}{ Particle \# } & \multirow{2}{*}{$\begin{array}{c}\text { Grid } \\
\text { Position }\end{array}$} & \multicolumn{3}{|c|}{ Diattenuation } & \multicolumn{3}{|c|}{ Equivalent $\mathrm{BAFO}=1+3 \mathrm{~N}$} \\
\hline & & Average & St. Dev. & Ave. Error & Average & St. Dev. & Ave. Error \\
\hline 1 & 4,4 & 0.1243 & 0.0118 & 0.0009 & 1.3729 & 0.0354 & 0.0027 \\
\hline 2 & 4,5 & 0.1253 & 0.0119 & 0.0009 & 1.3759 & 0.0357 & 0.0027 \\
\hline 3 & 4,6 & 0.1243 & 0.0135 & 0.0008 & 1.3729 & 0.0405 & 0.0024 \\
\hline 4 & 5,4 & 0.1310 & 0.0206 & 0.0011 & 1.3930 & 0.0618 & 0.0033 \\
\hline 5 & 5,5 & 0.1317 & 0.0150 & 0.0011 & 1.3951 & 0.0450 & 0.0033 \\
\hline 6 & 5,6 & 0.1376 & 0.0132 & 0.0011 & 1.4128 & 0.0396 & 0.0033 \\
\hline 7 & 6,4 & 0.1296 & 0.0182 & 0.0011 & 1.3888 & 0.0546 & 0.0033 \\
\hline 8 & 6,5 & 0.1371 & 0.0129 & 0.0010 & 1.4113 & 0.0387 & 0.0030 \\
\hline 9 & 6,6 & 0.1364 & 0.0145 & 0.0011 & 1.4092 & 0.0435 & 0.0033 \\
\hline 10 & 5,7 & 0.1246 & 0.0146 & 0.0011 & 1.3738 & 0.0438 & 0.0033 \\
\hline \multicolumn{2}{|c|}{ Average } & 0.1302 & 0.0146 & 0.0010 & 1.3906 & 0.0439 & 0.0031 \\
\hline
\end{tabular}

Mean of average BAFo per particle: 1.3906

Standard deviation of average BAFo per particle: 0.0164

\section{Comments}


Data Report Form DRF-18A: Measurement of Pyrocarbon Anisotropy using the 2-MGEM - IPyC

\begin{tabular}{|r|l|}
\hline Procedure: & AGR-CHAR-DAM-18 Rev. 1 \\
\hline Operator: & G. E. Jellison \\
\hline Mount ID: & M08092401 \\
\hline Sample ID: & NUCO425-06DTF \\
\hline Sample Description: & AGR-3/4 DTF Particle Qualification Batch \\
\hline Folder containing data: & IIMc-agr\AGR\2-MGEM\R08100201\} $\\
{\hline}$
\end{tabular}

\begin{tabular}{|c|c|c|c|c|c|c|c|}
\hline \multirow{2}{*}{ Particle \# } & \multirow{2}{*}{$\begin{array}{c}\text { Grid } \\
\text { Position }\end{array}$} & \multicolumn{3}{|c|}{ Diattenuation } & \multicolumn{3}{|c|}{ True $\mathrm{BAFO}=(1+\mathrm{N}) /(1-\mathrm{N})$} \\
\hline & & Average & St. Dev. & Ave. Error & Average & St. Dev. & Ave. Error \\
\hline 1 & 4,4 & 0.1243 & 0.0118 & 0.0009 & 1.2839 & 0.0308 & 0.0023 \\
\hline 2 & 4,5 & 0.1253 & 0.0119 & 0.0009 & 1.2865 & 0.0311 & 0.0024 \\
\hline 3 & 4,6 & 0.1243 & 0.0135 & 0.0008 & 1.2839 & 0.0352 & 0.0021 \\
\hline 4 & 5,4 & 0.1310 & 0.0206 & 0.0011 & 1.3015 & 0.0546 & 0.0029 \\
\hline 5 & 5,5 & 0.1317 & 0.0150 & 0.0011 & 1.3034 & 0.0398 & 0.0029 \\
\hline 6 & $5, \overline{6}$ & 0.1376 & 0.0132 & 0.0011 & 1.3191 & 0.0355 & 0.0030 \\
\hline 7 & 6,4 & 0.1296 & 0.0182 & 0.0011 & 1.2978 & 0.0480 & 0.0029 \\
\hline 8 & 6,5 & 0.1371 & 0.0129 & 0.0010 & 1.3178 & 0.0346 & 0.0027 \\
\hline 9 & 6,6 & 0.1364 & 0.0145 & 0.0011 & 1.3159 & 0.0389 & 0.0029 \\
\hline 10 & 5,7 & 0.1246 & 0.0146 & 0.0011 & 1.2847 & 0.0381 & 0.0029 \\
\hline \multicolumn{2}{|c|}{ Average } & 0.1302 & 0.0146 & 0.0010 & 1.2994 & 0.0387 & 0.0027 \\
\hline
\end{tabular}

Mean of average BAFo per particle: 1.2994

Standard deviation of average BAFo per particle: 0.0145

\section{Comments}

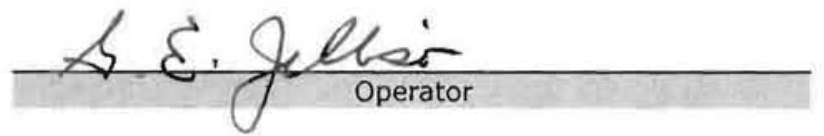

$$
(0-6-2008)
$$


Data Report Form DRF-22: Estimation of Average Particle Weight

\begin{tabular}{|r|l|}
\hline Procedure: & AGR-CHAR-DAM-22 Rev. 1 \\
\hline Operator: & Dixie Barker \\
\hline Particle Lot ID: & NUCO425-06DTF \\
\hline Particle Lot Description: & AGR-3/4 DTF Particle Qualification Batch \\
\hline Filename: & IImc-agr\AGRIParticleWeight।W08100802_DRF22R1.xIs \\
\hline
\end{tabular}

\begin{tabular}{|r|c|c|c|c|c|}
\hline & Sample 1 & Sample 2 & Sample 3 & Sample 4 & Sample 5 \\
\hline Weight of particles (g): & 0.1110 & 0.1193 & 0.1120 & 0.1164 & 0.1106 \\
\hline Number of particles: & 245 & 262 & 248 & 257 & 244 \\
\hline Average weight/particle (g): & $4.531 \mathrm{E}-04$ & $4.553 \mathrm{E}-04$ & $4.516 \mathrm{E}-04$ & $4.529 \mathrm{E}-04$ & $4.533 \mathrm{E}-04$ \\
\hline
\end{tabular}

Mean average weight/particle $(\mathrm{g}):[4.532 \mathrm{E}-04$

Standard error in mean average weight/particle $(\mathrm{g}): 6.00 \mathrm{E}-07$
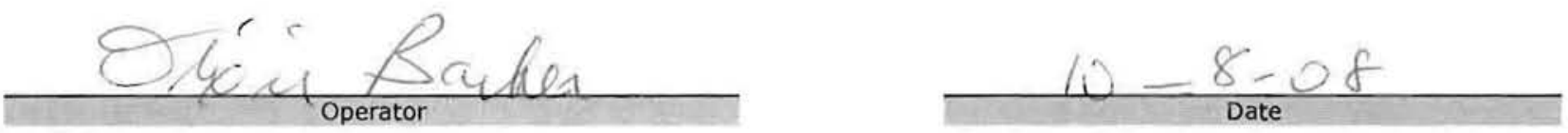


\section{Data Report Form DRF-31: Measurement of Open Porosity using a Mercury Porosimeter}

\begin{tabular}{|r|r|}
\hline Procedure: & AGR-CHAR-DAM-31 Rev. 1 \\
\hline Operator: & S. D. Nunn \\
\hline Coated particle batch ID: & NUCO425-06DTF-E01 \\
\hline Batch Description: & AGR-3/4 DTF Particle Qualification Batch \\
\hline Thermocouple Expiration Date: & $5 / 15 / 09$ \\
\hline Penetrometer Expiration Date: & $7 / 10 / 09$ \\
\hline Completed DRF Filename: & IImc-agr\AGRIPorosimeter IS08100601/S08100601_DRF31R1.xIS \\
\hline
\end{tabular}

Mean average weight/particle $(g): 4.53 E-04$

Standard error in mean average weight/particle $(g): 6.00 \mathrm{E}-07$

\begin{tabular}{|r|l|}
\hline Weight of particles (g): & 13.3968 \\
\hline Approximate number of particles: & 29560 \\
\hline Uncertainty In number of particles: & 39 \\
\hline Total envelope volume of sample (cc): & 1.625 \\
\hline Average envelope volume/particle (cc): & $5.50 \mathrm{E}-05$ \\
\hline Sample envelope density (g/cc): & 8.244 \\
\hline
\end{tabular}

Average particle diameter (microns): $4.72 \mathrm{E}+02$ Average surface area/particle $(\mathrm{cm} 2): 6.99 \mathrm{E}-03$

Total sample surface area $(\mathrm{cm} 2): 2.07 E+02$ Intruded mercury volume from $250-10,000$ psia (cc): $2.44 \mathrm{E}-02$ \begin{tabular}{l|l|l} 
Open porosity $(\mathrm{ml} / \mathrm{m} 2):$ & $1.18 \mathrm{E}+00$ & See note \\
\hline
\end{tabular}

\section{Comments}

The measured Intrusion volume and calculated open porosity was mostly a result of compression of the DTF coating rather than Intrusion into open pores. The actual open porosity is much lower and can not be determined with this method.

\section{S.D. Mun} Operator
$10 / 6 / 08$

Date




\section{Characterization of GA Archive DTF Particles}

Product inspection plan AGR-CHAR-PIP-08 was used as a guideline to characterize an archive sample of DTF particles. These particles were identified as having been produced by General Atomics (GA). An identification number of 8662-133 was on the label of the container of particles. This section contains the inspection report form (IRF-08) and associated data report forms resulting from that analysis.

Open porosity was not measured on this sample due to the limited number of particles available and the previous results on other DTF particles, which indicated that the measurement was not possible. Communication with John Saurwein at GA determined that no historical data was available on open porosity for these particles, and that such analysis was probably not performed. 


\section{Inspection Report Form IRF-08: AGR-3/4 Designed to Fail Coated Particle Batches}

Procedure: AGR-CHAR-PIP-08 Rev. 0

Coated particle batch ID: $8662-133$

Coated particle batch description: GA Archive DTF particles

\begin{tabular}{|c|c|c|c|c|c|c|c|c|c|}
\hline \multirow[b]{2}{*}{ Property } & \multicolumn{4}{|c|}{ Measured Data } & \multirow{2}{*}{$\begin{array}{c}\text { Specification } \\
\text { INL EDF-6638 } \\
\text { Rev. } 1 \\
\end{array}$} & \multirow[b]{2}{*}{ Acceptance Criteria } & \multirow{2}{*}{$\begin{array}{l}\text { Acceptance } \\
\text { Test Value }\end{array}$} & \multirow{2}{*}{$\begin{array}{l}\text { Pass } \\
\text { or } \\
\text { fail }\end{array}$} & \multirow{2}{*}{$\begin{array}{l}\text { Data } \\
\text { Record: }\end{array}$} \\
\hline & $\begin{array}{c}\text { Mean } \\
(x)\end{array}$ & $\begin{array}{l}\text { Std. Dev. } \\
\text { (s) }\end{array}$ & $\begin{array}{l}\text { a measured } \\
(n)\end{array}$ & $\begin{array}{l}k \text { or } t \\
\text { value }\end{array}$ & & & & & \\
\hline \multirow{3}{*}{$\begin{array}{c}\text { Average DTF thickness for } \\
\text { each particle }(\mu \mathrm{m})\end{array}$} & \multirow{3}{*}{20.2} & \multirow{3}{*}{1.0} & \multirow{3}{*}{72} & \multirow{2}{*}{1.667} & \multirow{2}{*}{$\begin{array}{c}\text { mean } \\
20 \pm 5\end{array}$} & $A=x-t s / V n \geq 15$ & 20.0 & pass & \multirow{3}{*}{$\begin{array}{l}\text { DRF-33 } \\
\text { DRF-34 }\end{array}$} \\
\hline & & & & & & $B=x+t s / \sqrt{n} \leq 25$ & 20.4 & pass & \\
\hline & & & & 2.753 & $\begin{array}{l}\text { dispersion } \\
\leq 0.01 \leq 8\end{array}$ & $C=x-k s>8$ & 17.4 & pass & \\
\hline \multirow{2}{*}{ DTF sink/float density $\left(\mathrm{Mg} / \mathrm{m}^{2}\right)$} & \multirow{2}{*}{1.9441} & \multirow{2}{*}{0.0064} & \multirow{2}{*}{25} & \multirow{2}{*}{2.711} & \multirow{2}{*}{$\begin{array}{c}\text { mean } \\
1.95=0.05\end{array}$} & $A=x-t s / V n \geq 1.90$ & 1.942 & pass & \multirow{2}{*}{ DRF-03 } \\
\hline & & & & & & $B=x+t s / \sqrt{n} \leq 2.00$ & 1.946 & pass & \\
\hline DTF anisotropy (BAFo equivalent) & 1.250 & 0.016 & 10 & 1.833 & \multicolumn{4}{|c|}{ Measurement Only } & DRF-18 \\
\hline DTF open porosity $\left(\mathrm{ml} / \mathrm{m}^{2}\right)$ & & & & & \multicolumn{4}{|c|}{ Measurement Only } & $\begin{array}{l}\text { DRF-22 } \\
\text { DRF-31 }\end{array}$ \\
\hline
\end{tabular}

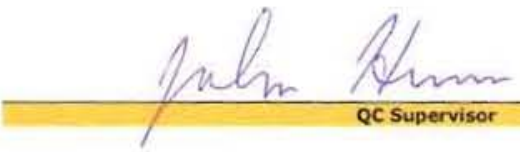

Accept coated particle batch (Yes or No):

\section{$10-22 \cdot 08$}

Date
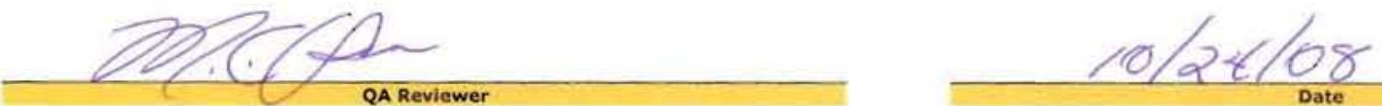
Data Reoort Form DRF-33: Imaging of Small Particle Cross-sections Using an Optical Microscove System

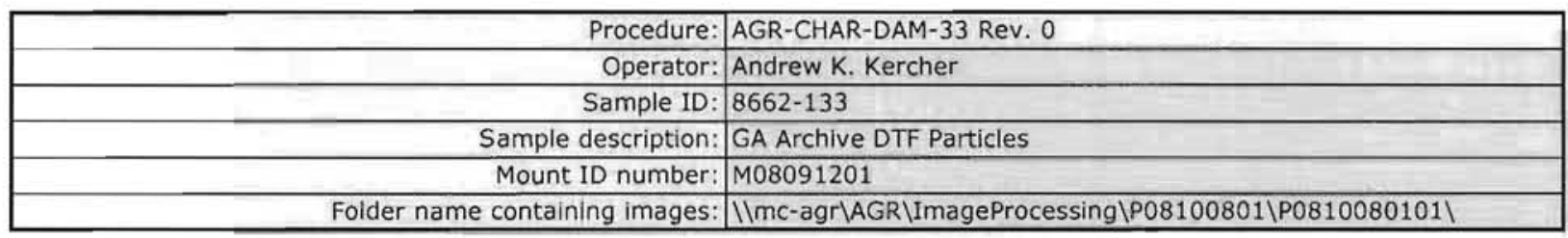

\begin{tabular}{|r|r|}
\hline DMR calibration expiration date: & $9 / 15 / 2009$ \\
\hline Calibrated pixels/micron: & 4.4833 \\
\hline Stage micrometer calibration expiration date: & $2 / 13 / 2009$ \\
\hline Measured value for $300 \mu \mathrm{m}$ in stage micrometer image $(\mu \mathrm{m}):$ & 299.8 \\
\hline
\end{tabular}

\begin{tabular}{|c|c|c|c|}
\hline \multicolumn{4}{|c|}{ Polish-down distance $n, m(\mu \mathrm{m})$} \\
\hline 2,2 & 2,8 & 8,2 & 8,8 \\
\hline \hline 169 & 166 & 162 & 157 \\
\hline
\end{tabular}

\section{Approximate layer width in polish plane ( $\mu \mathrm{m})$}

\begin{tabular}{|l|c|c|c|c|}
\hline Kernel radius & Layer 1 & Layer 2 & Layer 3 & Layer 4 \\
\hline \hline
\end{tabular}
169

$$
\text { October } 8,2008
$$




\section{Data Report Form DRF-34A: Measurement of Laver 1 Thickness}

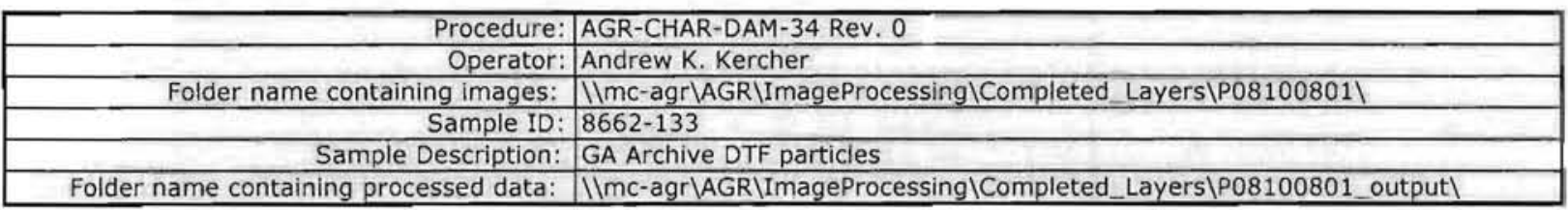

Number of layers analyzed: Mean of the average layer 1 thickness of each particle $(\mu \mathrm{m})$ : Standard deviation in the average layer 1 thickness of each particle $(\mu \mathrm{m})$ : 20.2

\section{Distribution of the average laver 1 thickness (top binned)}

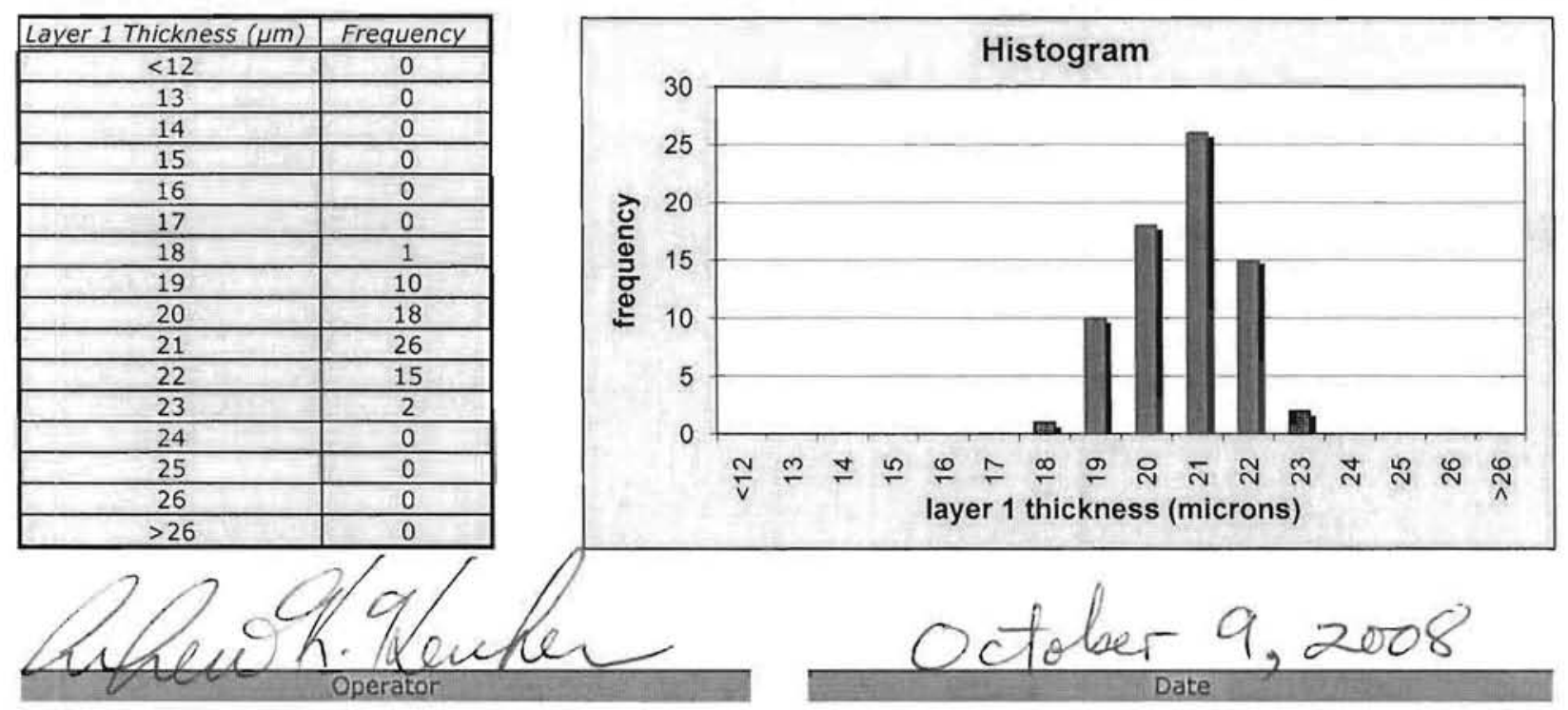


Data Report Form DRF-03: Measurement of PYC Density using a Density Gradient Column

\begin{tabular}{|r|l|}
\hline Procedure: & AGR-CHAR-DAM-03 Rev. 3 \\
\hline Operator: & Dixie Barker \\
\hline Filename: & IImc-agr\AGRIDensityColumn\D08100901_DRF03R3.xIs \\
\hline Sample ID: & $8662-133$ \\
\hline Sample description: & GA Archive DTF Particles \\
\hline Float expiration date: & $07 / 2012$ \\
\hline Gauge expiration date: & $11 / 2008$ \\
\hline Bath temperature: & $23.5{ }^{\circ} \mathrm{C}$ \\
\hline
\end{tabular}

\begin{tabular}{|c|c|c|c|}
\hline \multicolumn{4}{|c|}{ Calibrated Floats } \\
\hline Density & $\begin{array}{c}\text { Top of } \\
\text { Float }\end{array}$ & $\begin{array}{c}\text { Bottom of } \\
\text { Float }\end{array}$ & $\begin{array}{c}\text { Center of } \\
\text { Mass }\end{array}$ \\
\hline 1.800 & 97.71 & 106.10 & 101.91 \\
\hline 1.850 & 210.35 & 218.52 & 214.44 \\
\hline 1.900 & 320.91 & 326.88 & 323.90 \\
\hline 1.950 & 427.13 & 434.08 & 430.61 \\
\hline 2.000 & 525.13 & 532.00 & 528.57 \\
\hline
\end{tabular}

\begin{tabular}{|c|c|c|c|}
\hline \multicolumn{4}{|c|}{ Linear Fit } \\
\hline slope & StDev & intercept & StDev \\
\hline $4.67 \mathrm{E}-04$ & $2.82 \mathrm{E}-06$ & $1.75 \mathrm{E}+00$ & $9.76 \mathrm{E}-04$ \\
\hline
\end{tabular}

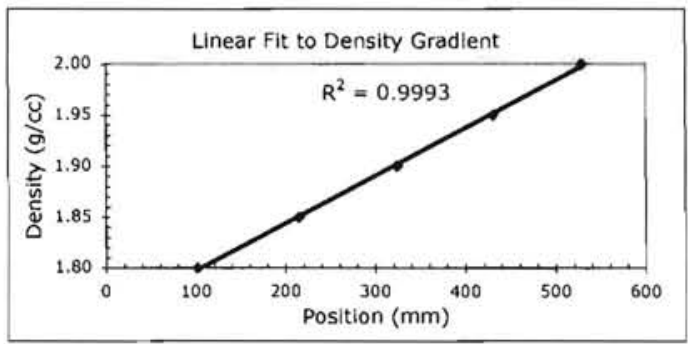

\begin{tabular}{|c|c|c|c|c|c|c|c|c|}
\hline \multicolumn{9}{|c|}{ Sample Density } \\
\hline $\begin{array}{l}\text { Fragment } \\
\text { Number }\end{array}$ & $\begin{array}{c}\text { Fragment } \\
\text { Position }\end{array}$ & $\begin{array}{l}\text { Calculated } \\
\text { Density }\end{array}$ & $\begin{array}{c}\text { Fragment } \\
\text { Number }\end{array}$ & $\begin{array}{l}\text { Fragment } \\
\text { Position }\end{array}$ & $\begin{array}{l}\text { Calculated } \\
\text { Density }\end{array}$ & $\begin{array}{l}\text { Fragment } \\
\text { Number }\end{array}$ & $\begin{array}{c}\text { Fragment } \\
\text { Position }\end{array}$ & $\begin{array}{c}\text { Calculated } \\
\text { Density }\end{array}$ \\
\hline 1 & 384.01 & 1.9300 & 26 & & & 51 & & \\
\hline 2 & 392.58 & 1.9340 & 27 & & & 52 & & \\
\hline 3 & 395.69 & 1.9354 & 28 & & & 53 & & \\
\hline 4 & 397.41 & 1.9362 & 29 & & & 54 & & \\
\hline 5 & 401.54 & 1.9381 & 30 & & & 55 & & \\
\hline 6 & 405.54 & 1.9400 & 31 & & & 56 & & \\
\hline 7 & 405.10 & 1.9398 & 32 & & & 57 & & \\
\hline 8 & 405.95 & 1.9402 & 33 & & & 58 & & \\
\hline 9 & 407.79 & 1.9411 & 34 & & & 59 & & \\
\hline 10 & 410.97 & 1.9426 & 35 & & & 60 & & \\
\hline 11 & 412.25 & 1.9432 & 36 & & & 61 & & \\
\hline 12 & 414.98 & 1.9444 & 37 & & & 62 & & \\
\hline 13 & 414.98 & 1.9444 & 38 & & & 63 & & \\
\hline 14 & 416.77 & 1.9453 & 39 & & & 64 & & \\
\hline 15 & 418.13 & 1.9459 & 40 & & & 65 & & \\
\hline 16 & 421.53 & 1.9475 & 41 & & & 66 & & \\
\hline 17 & 425.47 & 1.9493 & 42 & & & 67 & & \\
\hline 18 & 426.45 & 1.9498 & 43 & & & 68 & & \\
\hline 19 & 425.73 & 1.9495 & 44 & & & 69 & & \\
\hline 20 & 427.60 & 1.9503 & 45 & & & 70 & & \\
\hline 21 & 432.73 & 1.9527 & 46 & & & 71 & 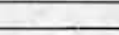 & \\
\hline 22 & 432.58 & 1.9527 & 47 & & & 72 & & \\
\hline 23 & 436.87 & 1.9547 & 48 & & & 73 & & \\
\hline 24 & 425.25 & 1.9492 & 49 & & & 74 & & \\
\hline 25 & 421.30 & 1.9474 & 50 & & & 75 & 4 & \\
\hline \multicolumn{4}{|c|}{ Average density of PyC fragments: } & \multicolumn{5}{|c|}{1.9441} \\
\hline \multirow{2}{*}{\multicolumn{4}{|c|}{ Standard deviation in density of PyC fragments: }} & \multicolumn{5}{|c|}{0.0064} \\
\hline & & & & \multicolumn{5}{|c|}{0.0016} \\
\hline
\end{tabular}
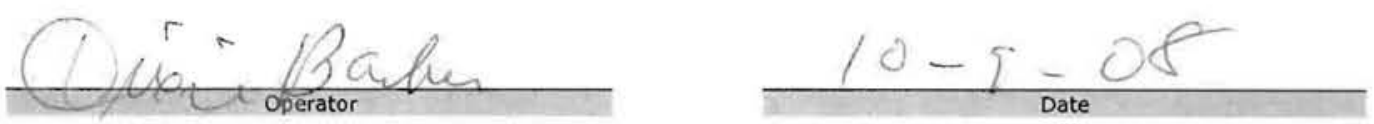
Data Report Form DRF-18A: Measurement of Pyrocarbon Anisotropy using the 2-MGEM - IPyC

\begin{tabular}{|r|l|}
\hline Procedure: & AGR-CHAR-DAM-18 Rev. 1 \\
\hline Operator: & G. E. Jellison \\
\hline Mount ID: & M08091201 \\
\hline Sample ID: & $8862-133$ \\
\hline Sample Description: & GA Archive DTF particles \\
\hline Folder containing data: & IIMc-agr\AGR\2-MGEM\R08091901 \\
\hline
\end{tabular}

\begin{tabular}{|c|c|c|c|c|c|c|c|}
\hline \multirow{2}{*}{ Particle \# } & \multirow{2}{*}{$\begin{array}{c}\text { Grid } \\
\text { Position }\end{array}$} & \multicolumn{3}{|c|}{ Diattenuation } & \multicolumn{3}{|c|}{ Equivalent $\mathrm{BAFO}=1+3 \mathrm{~N}$} \\
\hline & & Average & St. Dev. & Ave. Error & Average & St. Dev. & Ave. Error \\
\hline 1 & 4,4 & 0.0919 & 0.0124 & 0.0009 & 1.2757 & 0.0372 & 0.0027 \\
\hline 2 & 4,5 & 0.0851 & 0.0132 & 0.0010 & 1.2553 & 0.0396 & 0.0030 \\
\hline 3 & 4,6 & 0.0839 & 0.0138 & 0.0008 & 1.2517 & 0.0414 & 0.0024 \\
\hline 4 & 4,7 & 0.0920 & 0.0112 & 0.0008 & 1.2760 & 0.0336 & 0.0024 \\
\hline 5 & 5,4 & 0.0778 & 0.0167 & 0.0011 & 1.2334 & 0.0501 & 0.0033 \\
\hline 6 & 5,5 & 0.0775 & 0.0109 & 0.0010 & 1.2325 & 0.0327 & 0.0030 \\
\hline 7 & 5,6 & 0.0813 & 0.0111 & 0.0009 & 1.2439 & 0.0333 & 0.0027 \\
\hline 8 & 5,7 & 0.0813 & 0.0108 & 0.0009 & 1.2439 & 0.0324 & 0.0027 \\
\hline 9 & 6,5 & 0.0849 & 0.0160 & 0.0011 & 1.2547 & 0.0480 & 0.0033 \\
\hline 10 & 6,6 & 0.0791 & 0.0157 & 0.0010 & 1.2373 & 0.0471 & 0.0030 \\
\hline \multicolumn{2}{|c|}{ Average } & 0.0835 & 0.0132 & 0.0010 & 1.2504 & 0.0395 & 0.0029 \\
\hline
\end{tabular}

Mean of average BAFo per particle: 1.2504

Standard deviation of average BAFo per particle: 0.0157

\section{Comments}

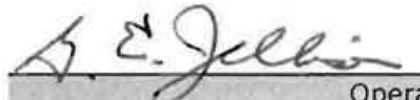


Data Report Form DRF-18A: Measurement of Pyrocarbon Anisotropy using the 2-MGEM - IPyC

\begin{tabular}{|r|l|}
\hline Procedure: & AGR-CHAR-DAM-18 Rev. 1 \\
\hline Operator: & G. E. Jellison \\
\hline Mount ID: & M08091201 \\
\hline Sample ID: & $8862-133$ \\
\hline Sample Description: & GA Archive DTF particles \\
\hline Folder containing data: & \Imc-agr\AGR\2-MGEM\R08091901\} $\\
{\hline}$
\end{tabular}

\begin{tabular}{|c|c|c|c|c|c|c|c|}
\hline \multirow{2}{*}{ Particle \# } & \multirow{2}{*}{$\begin{array}{c}\text { Grid } \\
\text { Position }\end{array}$} & \multicolumn{3}{|c|}{ Diattenuation } & \multicolumn{3}{|c|}{ True BAFo $=(1+\mathrm{N}) /(1-\mathrm{N})$} \\
\cline { 3 - 8 } & Average & St. Dev. & Ave. Error & Average & St. Dev. & Ave. Error \\
\hline \hline 1 & 4,4 & 0.0919 & 0.0124 & 0.0009 & 1.2024 & 0.0301 & 0.0022 \\
\hline 2 & 4,5 & 0.0851 & 0.0132 & 0.0010 & 1.1860 & 0.0315 & 0.0024 \\
\hline 3 & 4,6 & 0.0839 & 0.0138 & 0.0008 & 1.1832 & 0.0329 & 0.0019 \\
\hline 4 & 4,7 & 0.0920 & 0.0112 & 0.0008 & 1.2026 & 0.0272 & 0.0019 \\
\hline 5 & 5,4 & 0.0778 & 0.0167 & 0.0011 & 1.1687 & 0.0393 & 0.0026 \\
\hline 6 & 5,5 & 0.0775 & 0.0109 & 0.0010 & 1.1680 & 0.0256 & 0.0024 \\
\hline 7 & 5,6 & 0.0813 & 0.0111 & 0.0009 & 1.1770 & 0.0263 & 0.0021 \\
\hline 8 & 5,7 & 0.0813 & 0.0108 & 0.0009 & 1.1770 & 0.0256 & 0.0021 \\
\hline 9 & 6,5 & 0.0849 & 0.0160 & 0.0011 & 1.1856 & 0.0382 & 0.0026 \\
\hline 10 & 6,6 & 0.0791 & 0.0157 & 0.0010 & 1.1718 & 0.0370 & 0.0024 \\
\hline \multicolumn{2}{|c|}{ Average } & 0.0835 & 0.0132 & 0.0010 & 1.1822 & 0.0314 & 0.0023 \\
\hline
\end{tabular}

Mean of average BAFo per particle: 1.1822

Standard deviation of average BAFo per particle: 0.0125

\section{Comments}
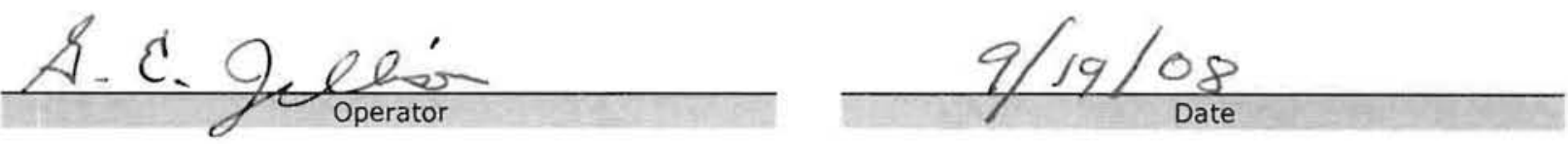